\title{
Proof of Security of Quantum Key Distribution With Two-Way Classical Communications
}

\author{
Daniel Gottesman and Hoi-Kwong Lo
}

\begin{abstract}
Shor and Preskill have provided a simple proof of security of the standard quantum key distribution scheme by Bennett and Brassard (BB84) by demonstrating a connection between key distribution and entanglement purification protocols (EPPs) with one-way communications. Here, we provide proofs of security of standard quantum key distribution schemes, BB84 and the six-state scheme, against the most general attack, by using the techniques of $t w o$-way entanglement purification. We demonstrate clearly the advantage of classical post-processing with two-way classical communications over classical post-processing with only one-way classical communications in quantum key distribution (QKD). This is done by the explicit construction of a new protocol for (the error correction/detection and privacy amplification of) BB84 that can tolerate a bit error rate of up to $18.9 \%$, which is higher than what any BB84 scheme with only one-way classical communications can possibly tolerate. Moreover, we demonstrate the advantage of the six-state scheme over BB84 by showing that the six-state scheme can strictly tolerate a higher bit error rate than BB84. In particular, our six-state protocol can tolerate a bit error rate of $26.4 \%$, which is higher than the upper bound of $25 \%$ bit error rate for any secure BB84 protocol. Consequently, our protocols may allow higher key generation rate and remain secure over longer distances than previous protocols. Our investigation suggests that two-way entanglement purification is a useful tool in the study of advantage distillation, error correction, and privacy amplification protocols.
\end{abstract}

Index Terms-Cryptography, key distribution, quantum cryptography, quantum information, quantum information processing.

\section{INTRODUCTION}

Q UANTUM key distribution (QKD) [2], [10 $]^{1}$ allows two parties to communicate in absolute privacy in the presence of an eavesdropper. Unlike conventional schemes of

Manuscript received July 8, 2001; revised August 16, 2002. The material in this paper was presented in part at the Sixth International Conference on Quantum Communication, Measurement and Computing (QCMC'02), Cambridge, MA, July 2002. Part of this work was performed while H.-K. Lo was at MagiQ Technologies, Inc., New York, NY 10001 USA.

D. Gottesman is with the Perimiter Institute for Theoretical Physics, Waterloo, ON N2J 2W9, Canada (e-mail: dgottesman@perimiterinstitute.ca). Part of this work was performed while he was with the Departmnent of Electrical Engineering and Computer Science, Computer Science Division, University of California, Berkeley, CA 94720 USA.

H.-K. Lo is with the Department of Electrical and Computer Engineering (ECE) and the Department of Physics, University of Toronto, Toronto, ON M5S 3G4 Canada (e-mail: hoi_kwong_lo@hotmail.com).

Communicated by P. W. Shor, Associate Editor for Quantum Information Theory.

Digital Object Identifier 10.1109/TIT.2002.807289

${ }^{1}$ The first paper on quantum cryptography was written by Stephen Wiesner around 1970, but it remained unpublished until 1983 [34]. For a survey on quantum cryptography, see, for example, [14]. For a review, see, for example, [12]. key distribution that rely on unproven computational assumptions, the security of QKD is guaranteed by the Heisenberg uncertainty principle of quantum mechanics. Much of the interest in QKD arises from the possibility of near-term real-life applications, whereas most other potential uses of quantum information remain remote. QKD has been performed experimentally over about $67 \mathrm{~km}$ of telecom fibers, and point-to-point through open air at a distance of about $23.4 \mathrm{~km}$. There are also proposals for key exchange from ground to satellites. (See [14], [12] for discussions.)

Today's technologies fall short of full control and manipulation of quantum states, so practical QKD protocols must use a much more restricted set of operations. The best known QKD protocol was published by Bennett and Brassard in 1984 [2]. The standard quantum key distribution scheme of Bennett and Brassard (BB84) is a simple "prepare-and-measure" protocol that can be implemented without a quantum computer (see [28], [26] for background on quantum computation). In a "prepare-and-measure" protocol, Alice simply prepares a sequence of single-photon signals and transmits them to Bob. Bob immediately measures those signals; thus, no quantum computation or long-term storage of quantum information is necessary, only the transmission of single-photon states, which can be performed through regular optical fibers. Therefore, "prepare-and-measure" schemes are good candidates for near-term implementations of quantum cryptography.

Of course, a theoretical description of a protocol is a mathematical idealization. Any real-life quantum cryptographic system is a complex physical system with many degrees of freedom, and is at best an approximation to the ideal protocol. Proving the security of any particular setup is a difficult task, requiring a detailed model of the apparatus. Even a seemingly minor and subtle omission can be fatal to the security of a cryptographic system.

Nevertheless, a number of important basic issues have been identified. See, for example, [27] for a discussion. For instance, the ideal theoretical version of BB84 uses a perfect single-photon source. It is important to know whether an eavesdropper can, in principle, exploit imperfect photon sources or other minor deviations from the ideal model (such as channel loss or detector dark counts). In this paper, we will not consider the issue of imperfections in the source or detectors. Instead, we will concentrate on the allowable bit error rate in the channel, and show that it can be at least $26.4 \%$ for a "prepare-and-measure" scheme.

To prove the security of a protocol, one must specify clearly what eavesdropping strategies are permissible. In classical cryptography, eavesdroppers are frequently given only a bounded 
amount of computation. Unfortunately, we do not, as yet, have a good grasp of what can be done with a short quantum computation, and provable bounds are elusive, even for classical computation. Other assumptions are similarly unreliable, so we resort to one of the most conservative assumptions, unconditional security - that is, security against the most general attacks allowed by quantum mechanics.

As it turned out, proving unconditional security even for an idealized system was very difficult. More than a decade passed between the original proposal for BB84 and the first general but rather complex proof of security by Mayers [32], [35], which was followed by a number of other proofs [5], [4]. Another approach to proving the security is to start by considering protocols which are less experimentally accessible. In particular, Lo and Chau [23], building on the quantum privacy amplification idea of Deutsch et al. [8], have proposed a conceptually simpler proof of security. However, the protocol proved secure has the unfortunate drawback of requiring a quantum computer. Recently, Shor and Preskill [33] have unified the techniques in [23] and [32], [35] and provided a simple proof of security of standard BB84. (See also [15] for a detailed exposition of this proof.) $)^{2}$

The idea of an entanglement purification protocol (EPP) [3] plays a key role in Shor and Preskill's proof. An EPP is a procedure allowing Alice and Bob to create a small number of reliable Einstein-Podolsky-Rosen (EPR) pairs from a larger number of noisy pairs. More specifically, Shor and Preskill consider schemes for entanglement purification with a classical side channel from Alice to Bob (one-way EPPs or 1-EPPs), which, by the earlier work of Bennett, DiVincenzo, Smolin, and Wootters (BDSW) [3], are mathematically equivalent to quantum error-correcting codes (QECCs).

As noted by BDSW, EPPs involving two-way communications between two parties can tolerate a substantially higher error rate than 1-EPPs. Those two-way EPPs (or 2-EPPs) are useful for the transmission of quantum signals, but not their storage in a noisy memory, since in a 2-EPP, the receiver Bob must send information to the sender Alice.

In this paper, we demonstrate that it is possible to create "prepare-and-measure" QKD schemes based on 2-EPPs, and that the advantages of 2-EPPs can survive. More specifically, we describe versions of BB84 and the six-state scheme [6] (another "prepare-and-measure" scheme) using two-way communcations and prove their security with allowed error rates substantially higher than any previous proofs.

Our results are significant for QKD for several reasons. First, our scheme can tolerate substantially higher bit error rates than all previous protocols. This may allow us to extend the distance of secure QKD and increase the key generation rate. Second, we demonstrate clearly the advantage of using two-way classical communications in the classical post-processing of signals in QKD. In particular, for both BB84 and the six-state scheme, our protocol tolerates a higher bit error rate than any one-way

\footnotetext{
${ }^{2}$ Mayers' and Shor-Preskill's proofs make different assumptions. While Mayers' proof assumes that Alice's preparation of the BB84 states is perfect, Shor and Preskill limit the types of imperfections allowed in Bob's measurement apparatus. A proof that takes into account more general imperfections remains to be published.
}

post-processing method. Third, our results show rigorously that the six-state protocol can tolerate a higher bit error rate than BB84. These facts can help direct experimentalists toward the most robust schemes for quantum key distribution.

There are good conceptual reasons as well for studying two-way QKD. The Shor and Preskill proof of security turns on the relationship between classical error correction and privacy amplification and QECCs. EPPs have a close relationship to QECCs, but the detailed connection between EPPs using one-way and two-way classical side channels is not well understood [3]; in fact, very little is known about 2-EPPs. A study of two-way QKD elucidates the relationship between the various aspects of quantum cryptography and 2-EPPs. It may help to spur progress in both the theoretical study of 2-EPPs and also their practical applications in a real experiment. This is so because "prepare-and-measure" QKD schemes, which we consider, can essentially be implemented in a real experiment [27]. Furthermore, the study of two-way QKD can clarify other proofs of security of QKD such as that due to Inamori [18], [19], and may make the connection to earlier studies of classical advantage distillation [29]-[31]. ${ }^{3}$

In Section II, we present the BB84 and six-state protocols and review known bounds on the bit error rates they tolerate. Section III reviews the necessary concepts from the theory of QECCs and EPPs. Even readers already familiar with these subjects may wish to read Sections II-C and III to acquaint themselves with our terminology. Section IV presents the Shor and Preskill proof of security. In Section V, we attempt a naive generalization of the proof to two-way protocols, which fails in an instructive way. In Section VI, we present the main theorem: EPPs satisfying the correct set of conditions can be made into secure "prepare-and-measure" QKD schemes with two-way communications. An example EPP satisfying the conditions is presented in Section VII; variations of this EPP produce the achievable error rates cited in this paper. We prove the main theorem in Section VIII.

\section{QKD PROTOCOLS AND BOUNDS ON PERFORMANCE}

\section{A. BB84 and the Six-State Scheme}

In the BB84 protocol for $\mathrm{QKD}$, Alice sends a qubit (i.e., a quantum bit or a two-level quantum system) in one of four states to Bob. The states $|0\rangle$ and $|+\rangle=(|0\rangle+|1\rangle / \sqrt{2}$ represent the classical bit 0 , while the states $|1\rangle$ and $|-\rangle=(|0\rangle-|1\rangle) / \sqrt{2}$ represent the bit 1 . Alice chooses one of these four states uniformly at random, and sends it to Bob, who chooses randomly to measure in either the $|0\rangle,|1\rangle$ basis (the " $Z$ " basis) or the $|+\rangle$, $|-\rangle$ basis (the " $X$ " basis). Then, Alice and Bob announce the basis each of them used for each state (but not the actual state sent or measured in that basis), and discard any bits for which

\footnotetext{
${ }^{3} \mathrm{An}$ important result in classical cryptography based on noisy channels is that a two-way side channel can actually increase the secrecy capacity of a noisy channel. That is, the secrecy capacity with a two-way side channel $C_{2}^{s}$ can be strictly greater than the secrecy capacity with only a one-way side channel $C_{1}^{s}$. See [29]-[31] for details. This is in sharp contrast with Shannon's channel coding theorem which states that two-way side channels do not increase channel capacity. The process of using two-way communications to share a secret in a way that is impossible with only one-way communications is called "advantage distillation."
} 
they used different bases. The remaining bits form the raw key, which will be processed some more to produce the final key.

The six-state protocol is quite similar, but Alice sends one of six states instead of one of four. The four states from BB84 are used (with the same meanings), plus the two states $(|0\rangle+$ $i|1\rangle) / \sqrt{2}$ and $(|0\rangle-i|1\rangle) / \sqrt{2}$, which represent 0 and 1 in the " $Y$ " basis. Bob chooses to measure randomly in the $X, Y$, or $Z$ basis, and again Alice and Bob discard any bits for which they used different bases. Thus, for the six-state scheme, the raw key consists of one-third of the qubits received on average, as opposed to one-half for BB84;4 however, as we shall see, the six-state scheme remains secure under noisier conditions.

Once they have produced the raw key, Alice and Bob select a sample of sufficient size (assume one-half the total raw key for simplicity), and publicly announce the values of those bits. They compare and calculate the fraction of bits which disagree; this is known as the "bit error rate." The bit error rate gives an estimate of the actual error rate for the remaining key bits. If the bit error rate is too high, Alice and Bob assume there is an eavesdropper and abort the protocol. Otherwise, Alice and Bob take their remaining bits and may correct them using a classical error-correcting code: that is, Alice announces her values for the parity checks of a classical linear code, and Bob compares his values for the same parity checks to deduce the locations of errors in the remaining key bits. He corrects those errors. Finally, Alice and Bob perform privacy amplification whose goal is to remove the eavesdropper's information on the final key: they choose some set of parities, and the final key bits are the values of those parities. After this procedure, provided the bit error rate is not too high, the final key is supposed to be secure against an eavesdropper Eve.

There are a few points about the protocols which deserve additional comment. First, all of Alice and Bob's classical communications occur over a public channel, so Eve also has available to her any information that was announced. However, the classical channel should be authenticated, so that Eve can only listen to it and not change it. Second, after producing the raw key and before performing the error test, Alice and Bob should agree on a random permutation to apply to their raw key bits. This simplifies the analysis, since Eve's attack under these circumstances might as well be symmetric over all qubits sent, and improves the tolerable bit error rate. Third, the meaning of "security" for this protocol is slightly subtle: for any attack chosen by Eve, either she will be detected, except with probability exponentially small in some security parameter $r$, or, with probability exponentially close to 1 , she will have an exponentially small amount of information, in some security parameter $s$, about the final key. A QKD scheme is efficient if the resources (in terms of the number of qubits sent, amount of computational power, etc.) required for its implementation are at most polynomial in the security parameters. For simplicity, it is quite common to take the security parameters to be $n$, the total number of qubits sent. As discussed in [25], other choices of the security parameters are perfectly acceptable.

${ }^{4}$ Prepare-and-measure QKD schemes can be made more efficient by employing a refined data analysis in which the bit error rates of the sampled data of the various bases are computed separately and each demanded to be small. See [25], [24] for discussions and a proof of the unconditional security of those efficient prepare-and-measure QKD schemes.
TABLE I

Bounds on the BIT ERRor RATE FOR BB84 AND THE SiX-STATE SCHEME Using ONE-WAY AND Two-WAY Classical Post-PROCESSING. THE LOWER BOUNDS FOR TWO-WAY POST-PROCESSING, 18.9\% FOR BB84 AND 26.4\% FOR THE SiX-STATE SCHEME, COME From THE CURRENT WORK

\begin{tabular}{ccc}
\hline \multicolumn{3}{c}{ BB84 } \\
& & \\
Upper bound & $14.6 \%$ & $1 / 4$ \\
Lower bound & $11.0 \%$ & $18.9 \%$ \\
& & \\
Six-state Scheme & \\
& & \\
& one-way & two-way \\
Upper bound & $1 / 6$ & $1 / 3$ \\
Lower bound & $12.7 \%$ & $26.4 \%$ \\
& & \\
\hline
\end{tabular}

\section{B. Known Bounds on the Performance of $Q K D$}

There are a number of upper and lower bounds known for the allowable bit error rate for these two protocols. In Table I, we summarize the bounds for BB84 and the six-state scheme. The tables give bounds for schemes that use one-way and two-way classical communications during the post-processing phase. The upper bounds are derived by considering some simple individual attacks, and determining when these attacks can defeat QKD. The lower bounds come from protocols that have been proved secure. For both BB84 and the six-state scheme, our new lower bounds for two-way classical post-processing schemes are substantially better than the upper bounds for schemes with one-way classical post-processing. Therefore, our results demonstrate clearly that our schemes can tolerate higher bit error rates than any possible schemes with only one-way classical post-processing can.

The upper bounds for one-way post-processing come from attacks based on optimal approximate cloning machines [11], [7], [1]. Although perfect cloning of an unknown quantum state is strictly forbidden by the uncertainty principle of quantum mechanics, approximate cloning is possible. Optimal approximate cloning has recently been experimentally demonstrated [21]. More specifically, Eve intercepts all of Alice's signals from the quantum channel. Using the appropriate optimal cloner, Eve can generate two equally good approximate copies of the original signal. In the case of BB84, the resulting bit error rate in a single copy is about $14.6 \%$ [11], [7], and it is $1 / 6$ for the six-state scheme [1]. Eve then keeps one copy herself and sends the second copy to Bob. With only one-way classical processing, Bob is not allowed to send classical signals to Alice. 5 Therefore, Bob and Eve are in a completely symmetric situation: if Bob can generate a key based on subsequent classical transmissions from Alice, Eve must be able to do the same. Therefore, at this error rate $(14.6 \%$ or $1 / 6)$, the QKD scheme must be insecure with one-way post-processing.

5 If one allows Bob to send classical messages to Alice only (but not from Alice to Bob), in the context of coherent state QKD, it is known that such backward one-way communications can actually help to beat the approximate cloning attack. However, the issue of unconditional security remains open. See [16] for details. 
The upper bounds for two-way post-processing come from an intercept and resend eavesdropping strategy. Eve intercepts each qubit sent by Alice. She chooses to measure in a random basis from the appropriate list $(X, Z$ for BB84 or $X, Y, Z$ for the six-state scheme). She records her measurement outcome and prepares a single photon in the polarization given by her measurement outcome and resends such a photon to Bob. Note that whatever Bob can do from this point on can be simulated by a classical random variable prepared by Eve, who has a classical record of it, and a local random number generator possessed by Bob. Therefore, secure QKD is impossible even with two-way classical communications between Alice and Bob. For BB84, the intercept and resend strategy gives an error rate of $25 \%$ : half the time Eve has chosen the correct basis, so there is no error, and half the time she has chosen the wrong basis, in which case there is a $50 \%$ chance of an error, for a net error rate of $1 / 4$. For the six-state scheme, intercept and resend gives an error rate of $1 / 3$ : Eve has the correct basis only $1 / 3$ of the time, and the remaining $2 / 3$ of the time, she has a $50 \%$ chance of introducing an error.

The lower bounds in Table 1 come from proofs of security. The Shor and Preskill proof shows that QKD with one-way communications can be secure with data rate at least $1-2 h(p)$, where $p$ is the bit error rate and

$$
h(x)=-x \log _{2} x-(1-x) \log _{2}(1-x)
$$

is the Shannon entropy. This reaches 0 when $p$ is about $11.0 \%$. For the six-state scheme, this result has been slightly improved by one of us (H.-K. Lo) [22] to allow secure QKD up to a bit error rate of about $12.7 \% .^{6}$ With two-way communications during post-processing, Shor and Preskill's result and Lo's result remain the best prior results. (Lo's result is marginally better than Inamori's result [19] for the six-state scheme, which requires two-way classical post-processing.) In this paper, we present significant improvements on both lower bounds.

\section{EPP Schemes for $Q K D$}

For our proof of security, it will be helpful to consider another class of scheme based on EPPs (which are described in more detail in Section III). For these QKD schemes, which we will refer to as EPP schemes or EPP protocols, ${ }^{7}$ Alice prepares a number of EPR pairs $\left|\Psi^{+}\right\rangle=(|00\rangle+|11\rangle) / \sqrt{2}$. On the second qubit of each pair, Alice then performs a random rotation chosen either from the set $I, H$ or the set $I, T, T^{2} . I$ is the identity operation, $H$ is the Hadamard transform, which swaps states in the $X$ and $Z$ bases, and $T$ is a unitary operation which takes states in the $X$ basis to the $Y$ basis, states in the $Y$ basis to the $Z$ basis, and states in the $Z$ basis to the $X$ basis.

We will refer to the first case (with $I$ and $H$ ) as the two-basis EPP protocol, and the second case (with $I, T$, and $T^{2}$ ) as the three-basis EPP protocol. The two-basis scheme will produce a

\footnotetext{
${ }^{6}$ The result in [22] makes use of the nontrivial mutual information between the bit-flip and phase error syndromes, and of the degenerate codes studied by DiVincenzo, Shor, and Smolin [9].

7"EPP protocol" sounds redundant since the second "P" in "EPP" also stands for "protocol." However, it is not really redundant, since the full phrase is short for "quantum key distribution protocol based on an entanglement purification protocol."
}

protocol related to $\mathrm{BB} 84$, while the three-basis scheme produces a protocol related to the six-state scheme. We can also consider efficient schemes in which the rotations are not performed with equal probabilities. These produce efficient BB84 and six-state schemes [25], [24], which have a higher rate of key generation per qubit transmitted.

After performing the rotation, Alice sends the second qubit of each pair to Bob. When Bob acknowledges receiving the transmission, Alice announces which rotation she performed for each pair. Bob reverses this rotation. Then Alice and Bob agree on a random permutation of the EPR pairs, and select a subset (half of the pairs by default) to measure (in the $Z$ basis) to test for errors. They compare the results of the test, and abort if the error rate is too high. If not, Alice and Bob perform an EPP to extract good entangled pairs. Then they measure (again in the $Z$ basis) the remaining pairs and use the result as their secret key.

The security proofs we review in Section IV show that the security of BB84 and the six-state scheme can be reduced to the security of the above EPP schemes using appropriate EPPs. The protocols that lead to traditional prepare-and-measure one-way post-processing schemes are EPPs using just one-way communications; in this paper, we present two-way postprocessing schemes that arise from EPPs with two-way classical communications.

\section{ENTANGLEMENT PURIFICATION AND QUANTUM ERROR CORRECTION}

Suppose Alice and Bob are connected by a noisy quantum channel (and perhaps also a noiseless classical channel). Entanglement purification provides a way of using the noisy quantum channel to simulate a noiseless one. More concretely, suppose Alice creates $N$ EPR pairs and sends half of each pair to Bob. If the quantum communication channel between Alice and Bob is noisy (but stationary and memoryless), then Alice and Bob will share $N$ imperfect EPR pairs, each in the state $\rho$. They may attempt to apply local operations (including preparation of ancillary qubits, local unitary transformations, and measurements) and classical communications (LOCCs) to purify the $N$ imperfect EPR pairs into a smaller number, $n$, EPR pairs of high fidelity. This process is called an EPP and was first studied by BDSW [3].

One way to classify EPPs is in terms of what type of classical communications they require. Fig. 1(a) shows the structure of EPPs that can be implemented with only one-way classical communications from Alice to Bob, known as 1-EPPs. Fig. 1(b) shows the structure of EPPs requiring two-way classical communications, known as 2-EPPs.

Typically, a 1-EPP will consist of Alice measuring a series of commuting operators and sending the measurement result to Bob. Bob will then measure the same operators on his qubits. If there is no noise in the channel, Bob will get the same results as Alice, but of course when noise is present, some of the results will differ. From the algebraic structure of the list of operators measured, Bob can deduce the location and nature of the errors and correct them. Unfortunately, the process of measuring EPR pairs will have destroyed some of them, so the resulting state consists of fewer EPR pairs than Alice sent. 


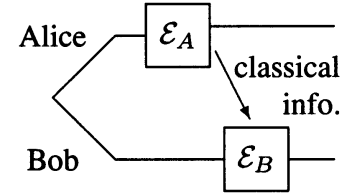

(a)

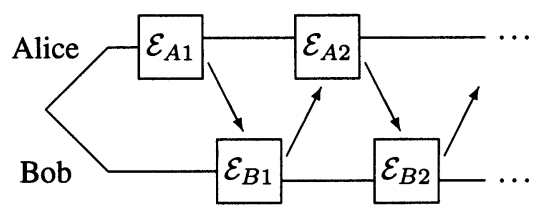

(b)

Fig. 1. (a) A 1-EPP. Alice performs some unitary operations and measurements, then makes a transmission to Bob, who performs another unitary transformation, possibly based on Alice's classical transmission. (b) A 2-EPP. Alice and Bob alternate local operations and classical transmissions. Each operation can depend on the contents of earlier transmissions. The procedure can extend indefinitely.

As noted by BDSW, a 1-EPP is mathematically equivalent to a QECC (see [13], [28] for background on QECCs). Instead of measuring a series of operators and transmitting the results, Alice instead encodes Bob's qubits into a particular predetermined eigenspace of the list of operators. Then when Bob receives the qubits, he can measure the same list of operators, telling him the error syndrome for the QECC given by that subspace. For instance, if the channel only produces bit-flip errors, Alice can encode Bob's state using a random coset of a classical linear code, and then Bob measures the parity checks for that code. He determines what error the channel introduced by calculating how the coset has changed since Alice's transmission.

2-EPPs can be potentially more complex, but frequently have a similar structure. Again, Alice and Bob measure a set of identical operators. Then they compare their results, discard some EPR pairs, and together select a new set of operators to measure. An essential feature of a 2-EPP is that the subsequent choice of measurement operators may depend on the outcomes of previous measurements. This process continues for a while until the remaining EPR pairs have a low enough error rate for a 1-EPP to succeed. Then, a 1-EPP is applied.

Unfortunately, not all EPPs are suitable for making a prepare-and-measure QKD protocol. The next few definitions are designed to set the stage for the detailed sufficient conditions in our main theorem. We will, for instance, primarily be interested in a restricted class of EPPs which involve the measurement of Pauli operators. The best studied EPPs can all be described in the "stabilizer" formulation, which employs Pauli operators extensively. Other EPPs might still be useful for QKD, but are less well studied.

Definition 1: A Pauli operator acting on $n$ qubits is a tensor product of individual qubit operators that are of the form $I$ (the identity)

$$
X=\left(\begin{array}{ll}
0 & 1 \\
1 & 0
\end{array}\right), \quad Y=\left(\begin{array}{cc}
0 & -i \\
i & 0
\end{array}\right), \quad \text { and } \quad Z=\left(\begin{array}{rr}
1 & 0 \\
0 & -1
\end{array}\right) \text {. }
$$

An $X$-type operator is a tensor product of just $I$ 's and $X$ 's, and a $Z$-type operator is a tensor product of just $I$ 's and $Z$ 's.
Note that the states in Section II described as being in the $X$, $Y$, or $Z$ bases are, in fact, eigenstates of the operators $X, Y$, and $Z$. A Calderbank-Shor-Steane (CSS) code involves measuring just $X$-type and $Z$-type Pauli operators. Also, note that any pair of $X, Y$, and $Z$ anticommute with each other (so, for instance, $X Z=-Z X)$. Finally, note that all Pauli operators have only eigenvalues +1 and -1 . Classical linear error-correcting codes can be understood as a measurement of a series of just $Z$-type operators: the eigenvalue of a $Z$-type operator is the parity of bits on which the operator acts as $Z$. (For instance, measuring $Z \otimes I \otimes Z$ gives the parity of the first and third bits.)

When dealing extensively with Pauli operations, it is helpful to also look at a more general class of operators which interact well with Pauli operations.

Definition 2: A unitary operation belongs to the Clifford group if it conjugates Pauli operators into other Pauli operators.

Thus, a Clifford group operation will map eigenstates of a Pauli operation into eigenstates of another Pauli operation. For instance, controlled-NOT (CNOT) and $H$ are both Clifford group operations. (In fact, the Clifford group is generated by CNOT, $H$, and the phase gate $|0\rangle \rightarrow|0\rangle,|1\rangle \rightarrow i|1\rangle$.)

Definition 3: We say an EPP (one-way or two-way) is symmetric if it can be described with a set of operators $\left\{M_{\mu}\right\}$, plus unitary decoding operations $U_{\mu} \otimes\left(P_{\mu} U_{\mu}\right)$. Each operator $M_{\mu}$ describes a measurement that may be made at some point in the protocol; the index $\mu$ describes a history of outcomes of earlier measurements as a string of 0 's and 1's. On the history $\mu$, Alice performs the measurement $M_{\mu}$ on her side, and Bob performs the measurement $M_{\mu}$ on his side. (They always perform the same sequence of measurements, thus the name "symmetric.") They then update the history $\mu$ by appending the parity of their two measurement outcomes ( 0 for the same outcome, 1 for opposite outcomes). The protocol begins with each person measuring the operator $M_{\emptyset}$. Each time the history is updated, Alice and Bob measure the operator corresponding to the new value of $\mu$, and again update the history according to the result. When there is no $M_{\mu}$ for the current history, Alice performs the operation $U_{\mu}$ and Bob performs the operation $P_{\mu} U_{\mu}$, and the protocol terminates.

Fig. 2 shows a symmetric EPP. See also Section III-A and Fig. 3 for another representation.

Note that if the history $\nu$ is an extension of the history $\mu$ (i.e., it is $\mu$ with additional bits appended), the operators $M_{\mu}, M_{\nu}$ should commute for the EPP to be realizeable using local operations and no additional resources. On the other hand, for two different extensions, $\nu_{1}$ and $\nu_{2}$, of the same history $\mu$, the corresponding operators $M_{\nu_{1}}$ and $M_{\nu_{2}}$ do not need to commute. This is because Alice and Bob never need to measure both operators for the same state.

For a 2-EPP, the commutation requirement is the only constraint on the $M$ 's. For a 1-EPP, we also require that the operators $M_{\mu}$ depend only on the length of $\mu$ (i.e., how many measurements have been made so far) and not the precise history. This is because in a 1-EPP, Alice cannot learn Bob's measurement outcomes and, therefore, cannot know the exact value of the history $\mu$. 


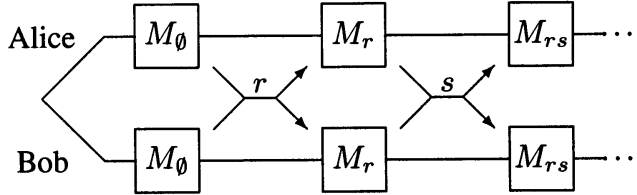

Fig. 2. Structure of a symmetric EPP. Alice and Bob measure the same sequence of operators. $r$ and $s$ are the parities of the outcomes of Alice's and Bob's measurements of $M_{\emptyset}$ and $M_{r}$, respectively.

The final operation $U_{\mu} \otimes P_{\mu} U_{\mu}$ serves two purposes. First, the measurements have determined a good deal of information about the state of the system, and we must disentangle that from the residual Bell states. Second, it acts to correct, discard, or otherwise eliminate any errors identified by the measurements. For instance, if the EPP locates pairs with errors, but does not identify what kind of errors are present, the final operation $U_{\mu}$ would likely permute the qubits to move the errors to a standard set of locations, which are then discarded. It is convenient to separate the decoding operation into two parts: $U_{\mu}$, which is performed by both people and represents decoding and discarding bad EPR pairs, and $P_{\mu}$, performed just by Bob, which represents correcting EPR pairs which will be kept. In practice, it is often easier to specify an EPP by including unitary operations in between measurements as well as at the end of the protocol, but this is an equivalent definition, since the measurement operators $M_{\mu}$ can instead be defined to take the change of basis into account. Notice that in a 1-EPP, $U_{\mu}$ cannot depend on $\mu$, whereas $P_{\mu}$ invariably will—otherwise, there would be no way to correct any errors discovered in the course of the protocol.

Definition 4: A symmetric EPP is a stabilizer EPP if all measurements $M_{\mu}$ are of eigenspaces of Pauli operations, the decoding operation $U_{\mu}$ is a Clifford group operation, and the correction operation $P_{\mu}$ is a Pauli operation. For a 1-EPP, we again make the restriction that $U_{\mu}=U$ is independent of $\mu$. A stabilizer EPP is CSS-like if all $M_{\mu}$ 's are $X$-type or $Z$-type Pauli operations, and $U_{\mu}$ involves only CNOTs.

Stabilizer 1-EPPs can be thought of as another guise of stabilizer QEECs. The measurements $M_{\mu}$ correspond to the generators of the code stabilizer. $U_{\mu}$ is the decoding operation, which for a stabilizer code is always from the Clifford group, and $P_{\mu}$ corrects the Pauli errors that have occurred. CSS-like 1-EPPs correspond to the class of CSS codes; since they are based on classical linear codes, the decoding only needs CNOT gates.

The same intuition applies to the case of 2-EPPs. The condition that decoding only needs CNOT means intuitively that the encoded $Z$ operation is, in fact, also of $Z$-type; that is, it can be written as a tensor product of $Z$ operators. The final correction operation $P_{\mu}$ is a Pauli operator because the error syndrome (disclosed in the two-way classical communication) should contain enough information to identify which Pauli error has occurred in the quantum channel.

The EPPs we will consider in this paper are all CSS-like EPPs. In fact, we will need to consider Alice and Bob choosing a random EPP out of a family of similar EPPs, but this does not produce any further intrinsic complications. For simplicity, we may describe EPPs that involve Clifford group or Pauli group operations in the middle of the series of measurements instead

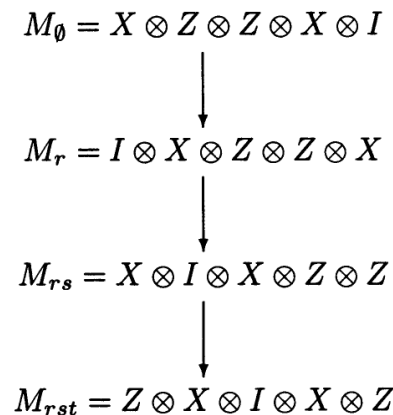

(a)

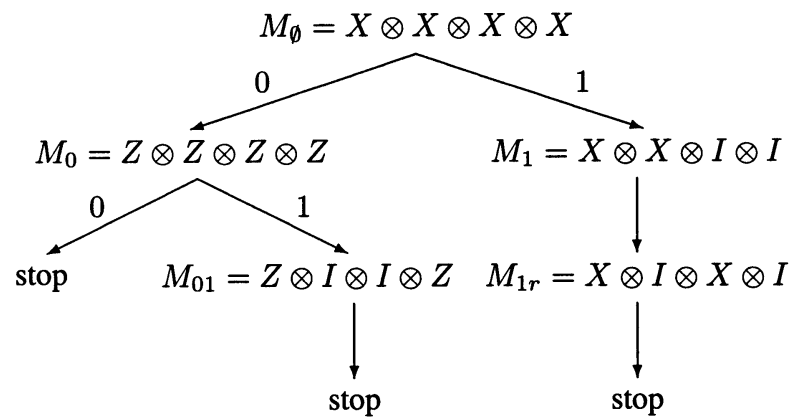

(b)

Fig. 3. (a) The tree diagram representation of a 1-EPP. The sequence of operators is fixed, so there is no branching. The 1-EPP shown corresponds to the 5-qubit QECC. (b) The tree diagram of a 2-EPP. Future operators may depend on the outcome of a measurement, allowing a branched tree. When the tree branches, edges are labeled by the outcome of the previous measurement. When it does not branch, no label is needed. Note that the tree does not need to branch uniformly, or even have uniform depth. The EPP in part (b) is CSS-like; the EPP in part (a) is not.

of the end, but this does not affect the definition at all; these EPPs can be rewritten to conform to the above definition of stabilizer or CSS-like EPPs.

\section{A. A Tree Diagram Representation}

The series of operators measured in a stabilizer 1-EPP or 2-EPP can be represented using a tree diagram representation. ${ }^{8}$ Each vertex is labeled by an operator $M_{\mu}$ that could be measured during the EPP. Each edge is labeled with one or more possible outcomes of the previous measurements. The edges are directed from the root of the tree (labeled by $M_{\emptyset}$ ) toward the leaves (labeled with $M_{\mu}$ for $\mu$ of maximal length), representing the time ordering of the measurements.

Given a tree diagram of the above form, we can read off the structure of the EPP. We start at the root of the tree, which is labeled by measurement $M_{\emptyset}$. We note the outcome and follow the edge which is labeled by that outcome. Then we perform the measurement which labels the new vertex, and follow the edge corresponding to the outcome of that measurement. We repeat this process until we reach the bottom of the tree, at which point we perform the appropriate unitary operation $U_{\mu} \otimes P_{\mu} U_{\mu}$. Each history $\mu$ corresponds to a path through the tree.

For any 1-EPP, the sequence of measurements does not depend on the outcome of any measurement. Therefore, a 1-EPP can be represented by a straight (directed) line (see Fig. 3(a)).

${ }^{8}$ We thank David DiVincenzo and Debbie Leung for suggesting the tree diagram representation. 
On the other hand, in a 2-EPP, the choice of measurement $M_{\mu}$ at any step $i$ can depend on the outcome of an earlier measurement $M_{\nu}$. This corresponds to a branch in the tree at step $i$ (see Fig. 3(b)).

\section{The Shor And Preskill Security Proof}

Next, we give the Shor and Preskill proof [33] of security of BB84. See also [15] for a more detailed version. Shor and Preskill's proof begins by following Lo and Chau's proof [23] of the security of a scheme using EPPs, and then shows that the security of BB84 follows from the security of the EPP scheme.

As noted before, in the EPP scheme, Alice creates $N$ EPR pairs and sends half of each to Bob. Alice and Bob then test the error rates in the $X$ and $Z$ bases on a randomly chosen subset of $m$ pairs. If the error rate is too high, they abort; otherwise, they perform an EPP $C$ on the remaining $N-m$ pairs. Finally, they measure (in the $Z$ basis) each of the $n$ EPR pairs left after $C$, producing a shared random key about which, they hope, Eve has essentially no information.

\section{A. Noisy Quantum Channels and Eavesdropping Strategies}

All of the QKD protocols we consider will take place over a noisy quantum channel, even when there is no eavesdropper present. We shall be primarily interested in a special class of quantum channels known as Pauli channels.

Definition 5: A quantum channel is any superoperator which acts on transmitted qubits. A Pauli channel $\left(\mathcal{P}_{i}, q_{i}\right)$ applies the Pauli operation $\mathcal{P}_{i}$ with probability $q_{i}$ (so we require $\sum q_{i}=1$ ). An uncorrelated Pauli channel $\left(q_{X}, q_{Y}, q_{Z}\right)$ applies a random Pauli operator independently on each qubit sent through the channel. It applies $X$ with probability $q_{X}, Y$ with probability $q_{Y}, Z$ with probability $q_{Z}$, and $I$ with probability $1-q_{X}-$ $q_{Y}-q_{Z}$

From the perspective of Alice and Bob, noise in the channel could have been caused by an eavesdropper Eve. We will need to consider two types of eavesdropping strategy by Eve. The first strategy, the joint attack, is the most general attack allowed by quantum mechanics.

Definition 6: In a joint attack by Eve, Eve has a quantum computer. She takes all quantum signals sent by Alice and performs an arbitrary unitary transformation involving those signals, adding any additional ancilla qubits she cares to use. She keeps any part of the system she desires and transmits the remainder to Bob. She listens to the public discussion (for error correction/detection and privacy amplification) between Alice and Bob before finally deciding on the measurement operator on her part of the system.

The joint attack allows Eve to perform any quantum operation on the qubits transmitted by Alice. For the security proof, we shall also consider a Pauli attack.

Definition 7: A Pauli attack by Eve is a joint attack where the final operation performed on the transmitted qubits is a general Pauli channel.

\section{B. EPP Protocols are Secure}

In this subsection, we will show that the EPP protocols described in Section II are secure. The argument is essentially that of [23]. First, what do we mean by "secure?"

Definition 8: A QKD protocol to generate $n$ key bits is correct if, for any strategy used by Eve, either Alice and Bob will abort with high probability or, with high probability, Alice and Bob will agree on a final key $k$ which is chosen nearly uniformly at random. The protocol is secure if, for any strategy used by Eve, either Alice and Bob will abort with high probability or Eve's information about the key will be at most $\exp (-s)$ for some security parameter $s$. In all cases, "with high probability" means with probability at least $1-\exp (-r)$ for some security parameter $r$. The resources required for the implementation of a QKD scheme must be at most polynomial in $r$ and $s$. For simplicity, in what follows, we will consider the case where $r=s$ and call it simply the security parameter.

Naively, one might consider a security requirement of the form $I_{\text {eve }}<\delta n$, where $I_{\text {eve }}$ is the eavesdropper's mutual information with the final key and $n$ is the length of the final key. However, such a definition of security is too weak, since it allows Eve to learn a few bits of a long message. For instance, the eavesdropper may know something about the structure of the message that Alice is going to send to Bob. Imagine that the last few characters of the message contain the password for launching a nuclear missile. In that case, Eve could compromise the security of the message by concentrating her information on the last few bits.

Another naive definition of security would be to require that $I_{\text {eve }}<e^{-\alpha n}$ for any eavesdropping strategy. Unfortunately, such a definition of security is too strong to be achievable. For instance, Eve can simply replace the signal prepared by Alice by sending Bob some signals with specific polarizations prepared by herself. Such an eavesdropping attack is highly unlikely to pass the verification test (by producing a small error rate). However, in the unlikely event that it does pass the verification test, Eve will have perfect information on the key shared between Alice and Bob, thus violating the security requirement $I_{\text {eve }}<e^{-\alpha n}$.

In fact, even the definition we give is probably not strong enough for some purposes: Eve can retain a quantum state at the end of the protocol, and the security definition should refer to that rather than bounding her classical information about the key. For instance, a better definition is: for any eavesdropping strategy, either Eve will almost surely be caught, or, for any two final values of the key, Eve's residual density matrices after the protocol concludes will have high fidelity to each other. That is, Eve cannot reliably distinguish between any pair of values of the key. We do not prove the stronger definition in this paper.

The question of defining security for quantum cryptography in a way that enables us to prove composibility of protocols remains an important open problem. For this paper, however, we simply use Definition 8.

Our method will be to relate the security of BB84 and the sixstate scheme to the security of EPP schemes, and we wish to say 
that when the EPP schemes are secure, so are the "prepare-andmeasure" schemes.

Definition 9: Suppose QKD protocol $\beta$ is correct and secure, with a security parameter $p$. Then QKD protocol $\alpha$ is said to have security similar to protocol $\beta$ when $\alpha$ is also correct and secure, and its security parameter $q$ is polynomially related to $p$. Furthermore, protocol $\alpha$ should abort at a given bit error rate only if protocol $\beta$ also aborts at that bit error rate.

To prove the security of EPP protocols, we first observe that we need only show Alice and Bob can generate states close to $n$ EPR pairs. This is a consequence of the following lemma (originally [23, Note 28]).

Lemma 1: If $\rho$ has a high fidelity $1-2^{-l}$ (for large $l$ ) to a state of $n$ perfect EPR pairs and Alice and Bob measure along a common axis to generate an $n$-bit key from $\rho$, then Alice and Bob will most likely share the same key, which is essentially random. Moreover, Eve's mutual information with the final key is bounded by $2^{-c}+O\left(2^{-2 l}\right)$, where

$$
c=l-\log _{2}\left[2 n+l+\left(1 / \log _{e} 2\right)\right] .
$$

In other words, Eve's information is exponentially small as a function of $l$.

The proof is given in Appendix I. The next step is to restrict our attention to Pauli attacks.

Lemma 2 [23]: Consider a stabilizer EPP protocol for QKD. Given any joint attack $\mathcal{A}$ by Eve, there is a Pauli attack for which the final density matrix $\rho_{\mathrm{AB}}$ of Alice and Bob has the same fidelity to $n$ EPR pairs, and which gives the same chance of having the QKD protocol abort.

We will only prove Lemma 2 for EPP protocols based on stabilizer EPPs, but the result holds for any EPP designed to correct Pauli channels (see [15] for the general proof). Pauli channels play a special role in the above lemma because most known QECCs (stabilizer codes, for instance) are designed to correct Pauli errors.

Proof: First, note that for a symmetric EPP, it would suffice if Alice and Bob had a way of measuring $M_{\mu} \otimes M_{\mu}$ directly instead of separately measuring $M_{\mu}$ on Alice's side and again on Bob's side. This is because all decisions are based on the parity of Alice's and Bob's results, which is equal to the eigenvalue of $M_{\mu} \otimes M_{\mu}$. Also, note that the EPR pair $\left|\Psi^{+}\right\rangle=$ $(|00\rangle+|11\rangle) / \sqrt{2}$ is a +1 eigenstate of the Pauli operators $X \otimes X$ and $Z \otimes Z$. (It is actually a -1 eigenstate of $Y \otimes Y$.)

Thus, let $W_{r}$ be a Bell measurement for the $r$ th EPR pair-a measurement of both $X \otimes X$ and $Z \otimes Z$. For a stabilizer EPP, $W_{r}$ commutes with $M_{\mu} \otimes M_{\mu}$ for all $\mu, r$ (note that each $M_{\mu}$ is likely to involve more than one EPR pair). Thus, if Alice and Bob first measure all the operators $M_{\mu} \otimes M_{\mu}$ and then measure $W_{r}$ for all $r$ after the EPP is concluded, the result is the same as if they first measured $W_{r}$ and then $M_{\mu} \otimes M_{\mu}$. Since they do not need the results of the measurements $W_{r}$, it is again equivalent if Eve measures $W_{r}$ instead of Alice and Bob.

That is, the following two situations are the same: a) Eve performs her attack $\mathcal{A}$ and then Alice and Bob measure $M_{\mu} \otimes M_{\mu}$, and b) Eve performs $\mathcal{A}$, measures $W_{r}$, and then Alice and Bob measure $M_{\mu} \otimes M_{\mu}$. By the argument of the previous paragraph, the attack in b) produces a density matrix with the same fidelity to $n$ EPR pairs as the attack in a). The attack $\mathcal{A}$ followed by measurement of $W_{r}$ is a Pauli attack. The initial state is a Bell state (the tensor product of $\left|\Psi^{+}\right\rangle$for all pairs), and the final state is a mixture of tensor products of Bell states (the outcome of measuring $W_{r}$ for each pair $r$ ). Each tensor product $\left|\Phi_{j}\right\rangle$ of Bell states can be associated with the unique Pauli operation $\mathcal{P}_{j}$ that maps $\left|\Psi^{+}\right\rangle^{\otimes N}$ to $\left|\Phi_{j}\right\rangle$, so Eve's attack is $\left(\mathcal{P}_{j}, q_{j}\right)$, where $q_{j}$ is the probability of getting the outcome $\left|\Phi_{j}\right\rangle$. Therefore, the lemma holds for a hypothetical protocol in which Alice and Bob measure $M_{\mu} \otimes M_{\mu}$ directly.

Of course, Alice and Bob have no way of doing this, so instead they must measure $M_{\mu}$ separately and compare results (with one- or two-way communications, as appropriate). Since this gives them more information, it certainly cannot help Eve. On the other hand, they do not actually use that information-from the definition of a symmetric EPP, only the relative measurement outcome between Alice and Bob matters. Therefore, having Alice and Bob measure $M_{\mu} \otimes M_{\mu}$ together produces the same fidelity and chance of aborting as when they measure $M_{\mu}$ separately.

This lemma is described in [23] as a "classicalization" or "quantum-to-classical reduction" because it reduces Eve's general quantum attack to a Pauli attack, which is classical in the sense that it can be described by classical probability theory. Lemma 2 allows us to simplify our discussion to just Pauli channels $\left(\mathcal{P}_{i}, q_{i}\right)$.

We can simplify further by taking into account the symmetry of the QKD protocol. Note that in the EPP protocols we described, Alice and Bob permute their qubits randomly before doing any other operations. So we may as well assume $q_{i}=q_{j}$ whenever $\mathcal{P}_{i}$ is a permutation of $\mathcal{P}_{j}$. That is, the attack is symmetric on the EPR pairs. Similarly, in the two-basis scheme, Alice performs randomly one of the two operations $I$, $H$, which produces a symmetry between the $X$ and $Z$ bases, so we can also assume $q_{i}=q_{j}$ whenever $\mathcal{P}_{i}$ is related to $\mathcal{P}_{j}$ by the Hadamard transform on any number of qubits. In the three-basis scheme, we can assume $q_{i}=q_{j}$ when $\mathcal{P}_{i}$ and $\mathcal{P}_{j}$ are related by $T$ or $T^{2}$ on some set of qubits.

Now, in the EPP protocols, Alice and Bob measure a random subset of $m$ qubits to test the error rate. From this, they are supposed to figure out what sort of Pauli channel the system has undergone. If the noise occurs independently on each qubit, this is just a straightforward problem in statistical inference. Of course, an eavesdropper need not use such a simple attack, but the symmetries of the protocol still allow Alice and Bob to make a good guess as to the true channel. For one thing, Eve has no way to distinguish between the test bits and the key bits, so the error rate measured for the test bits should be representative of the error rate on the key bits. What's more, Alice and Bob learn a good deal about the basis dependence of the channel as well.

Let us first consider the two-basis case more carefully. Suppose Alice and Bob find there are $p_{I} m_{I}$ errors among the $m_{I}$ qubits for which Alice did the operation $I$; these represent $X$ and $Y$ Pauli errors introduced by Eve. Similarly, 
they find $p_{H} m_{H}$ errors in the $m_{H}$ qubits for which Alice did the operation $H$; these represent $Y$ and $Z$ errors introduced by Eve. If this channel were an uncorrelated Pauli channel $\left(q_{X}^{0}, q_{Y}^{0}, q_{Z}^{0}\right)$, on average, we would expect $p_{I}=q_{X}^{0}+q_{Y}^{0}$ and $p_{H}=q_{Y}^{0}+q_{Z}^{0}$. In fact, if we consider the effective error rates after undoing the $I, H$ operations, we find $q_{X}=\left(q_{X}^{0}+q_{Z}^{0}\right) / 2$ and $q_{Z}=\left(q_{Z}^{0}+q_{X}^{0}\right) / 2$ because $I$ and $H$ are equally likely. That is, $q_{X}=q_{Z}$. The effective $Y$ error rate $q_{Y}=q_{Y}^{0}$.

Note that in the two-basis case, Alice and Bob are unable to deduce the most likely values of $q_{X}, q_{Y}$, and $q_{Z}$; they can only learn $p_{X}=q_{Y}+q_{Z}$ and $p_{Z}=q_{X}+q_{Y}$. Given the symmetry between $I$ and $H$, they, in fact, have $p_{X}=p_{Z}=\left(p_{I}+p_{H}\right) / 2$, but our discussion will keep $p_{X}$ and $p_{Z}$ as separate parameters. This allows most of our results to also apply to the efficient case [25], [24], where $I$ and $H$ have different probabilities.

The fact that Alice and Bob cannot completely learn the characteristics of even an uncorrelated Pauli channel suggests that it might be helpful to measure in more bases. This is the advantage of the six-state scheme, which is related to the three-basis EPP protocol. In that case, Alice and Bob measure $p_{I}, p_{T}, p_{T^{2}}$. For an uncorrelated Pauli channel $\left(q_{X}^{0}, q_{Y}^{0}, q_{Z}^{0}\right), p_{I}=q_{X}^{0}+q_{Y}^{0}$, $p_{T}=q_{Y}^{0}+q_{Z}^{0}$, and $p_{T^{2}}=q_{X}^{0}+q_{Z}^{0}$. Given the symmetry of the problem, after undoing the rotations, we get

$$
q_{X}=q_{Y}=q_{Z}=\left(q_{X}^{0}+q_{Y}^{0}+q_{Z}^{0}\right) / 3=\left(p_{I}+p_{T}+p_{T^{2}}\right) / 6 .
$$

Again, our discussion will allow $q_{X}, q_{Y}$, and $q_{Z}$ to be different to accommodate the efficient six-state protocol.

Given the error test, Alice and Bob deduce some values either for $p_{X}, p_{Z}$ or for all three quantities $q_{X}, q_{Y}, q_{Z}$. However, the error rate on the tested bits is only close to the error rate on the data bits. Therefore, they should use an EPP that is flexible enough to correct slightly more or less noisy Pauli channels than indicated by the test. In particular, when they deduce $q_{X}, q_{Y}$, and $q_{Z}$, they should perform an EPP capable of correcting any Pauli channel $\left(q_{X}^{t}, q_{Y}^{t}, q_{Z}^{t}\right)$ with $\left|q_{i}^{t}-q_{i}\right|<\epsilon$ for $i=X, Y, Z$ and some small $\epsilon$. Further, we should assume that, for any $\epsilon$, the fidelity of the final state to $n$ EPR pairs is exponentially close to 1 in $N$.

When Alice and Bob only learn $p_{X}$ and $p_{Z}$, they should allow additional flexibility for the value of $q_{Y}^{t}$. That is, their EPP should correct any Pauli channel $\left(p_{Z}^{t}-a, a, p_{X}^{t}-a\right)$ (with all three parameters nonnegative), again with $\left|p_{i}^{t}-p_{i}\right|<2 \epsilon$, for $i=X, Z$. Provided Alice and Bob use such an EPP, the next lemma says that the error test works and allows them to correct any symmetric Pauli channel, not just an uncorrelated one.

Lemma 3: Suppose the $N$ EPR pairs experience a Pauli channel $\left(\mathcal{P}_{i}, q_{i}\right)$ which is symmetric over the $N$ pairs, and that they use an EPP which corrects for any error rate close to those shown by the test bits, as described above. Then either they abort with high probability, or the final state has fidelity exponentially close to 1 in $N$ to the state of $n$ EPR pairs.

Since we only need to consider Pauli channels, the proof is just an exercise in classical probability, and is given in Appendix II.

From Lemmas 2 and 3, we know that for the EPP protocols we consider, given any strategy for Eve, either she has a large chance of getting caught, or the final state will have high fidelity to $n$ EPR pairs. Combining that with Lemma 1, we have shown the following.

Theorem 4: The EPP protocols for QKD are secure and correct.

\section{Prepare-and-Measure Protocols are Secure}

Given Theorem 4, Shor and Preskill [33] showed that one can prove the security of BB84. The same technique can be applied to show the security of the six-state scheme [22]. These two results can be combined into the following theorem.

Theorem 5-[33]: Given a QKD protocol based on a CSSlike 1-EPP, there exists a "prepare-and-measure" QKD protocol with similar security. That is, for any strategy by Eve to attack the "prepare-and-measure" protocol, there exists a strategy to attack the EPP protocol with similar probability of causing the protocol to abort and similar information gain to Eve if it does not abort. (Similar here means that the security parameters are polynomially related.)

Proof: The reduction to a "prepare-and-measure" protocol is done as a series of modifications to the EPP protocol to produce equivalent protocols. The main insight is that the $X$-type measurements do not actually affect the final QKD protocol, and therefore are not needed. The $X$-type measurements give the error syndrome for phase $(Z)$ errors, which do not affect the value of the final key. Instead, $Z$ errors represent information Eve has gained about the key. The phase information thus must be delocalized, but need not actually be corrected. The upshot is that Alice and Bob need not actually measure the $X$-type operators and can therefore manage without a quantum computer. Our initial goal is to manipulate the EPP protocol to make this clear. The $X$-type measurements do not, however, disappear completely: instead, they become privacy amplification.

For the first step, we modify the EPP to put it in a standard form. Because it is a CSS-like 1-EPP, there is no branching in the tree diagram, and each operator being measured is either $X$-type or $Z$-type. The operators all commute, and do not depend on the outcome of earlier measurements, so we can reorder them to put all of the $Z$-type measurements before all of the $X$-type measurements. Let us recall Definition 4 for a CSS-like 1-EPP. Now we have an EPP consisting of a series of $Z$-type measurements, followed by a series of $X$-type measurements, followed by CNOTs and Pauli operations (which we can represent as $I$, $X$, and/or $Z$ on each qubit). Then Alice and Bob measure all qubits in the $Z$ basis.

As a second step, we can move all $X$ Pauli operations to before the $X$-type measurements, since they commute with each other. Moreover, if Alice and Bob are simply going to measure a qubit in the $Z$ basis, there is no point in first performing a $Z$ phase-shift operation, since it will not affect at all the distribution of outcomes of the measurement.

We now have an EPP protocol consisting of $Z$-type measurements, followed by $X$ Pauli gates, followed by $X$-type measurements, followed by a sequence of CNOT gates which does not depend on the measurement outcomes. But nothing in the 
current version of the protocol depends on the outcomes of the $X$-type measurements, so those measurements are useless. We might as well drop them. Furthermore, $X$ Pauli operations and CNOT gates are just classical operations, so we might as well wait to do them until after the $Z$ basis measurement, which converts the qubits into classical bits.

What's more, it is redundant to perform $Z$-type measurements followed by measurement of $Z$ for each qubit. We can deduce with complete accuracy the outcome of each $Z$-type measurement from the outcomes of the measurements on individual qubits. For instance, if a sequence of three bits is measured to have the value 101, then we know that measurement of $Z_{1} \otimes Z_{2} \otimes Z_{3}$ will give the result +1 , as the parity of the three bits is even.

Thus, we are left with the following protocol: Alice prepares a number of EPR pairs, and sends half of each to Bob. She and Bob each perform the correction rotation ( $I$ or $H$ for the two-basis scheme; $I, T$, or $T^{2}$ for the three-basis scheme), then measure each qubit in the $Z$ basis. They use some of the results to test the error rate, and on the rest they perform some classical gates derived from the original EPP.

In fact, since Alice can perform her rotation and measurement before sending any qubits to Bob, she need not actually prepare entangled states. Instead, she simply generates a random number, which corresponds to the outcome of her $Z$-basis measurement, and sends Bob the state to which the EPR pair would have collapsed, given that measurement result. That is, she sends him $|0\rangle$ or $|1\rangle$ rotated by the appropriate gate $\left(I, H, T\right.$, or $\left.T^{2}\right)$. Bob inverts the rotation and measures.

Then they perform classical gates. To understand which gates, it is helpful to look more closely at the original EPP. When the EPP is based on a CSS code, the $Z$-type operators correspond to the parity checks of a classical error-correcting code $C_{1}$, and the $X$-type operators correspond to the parity checks of another classical code $C_{2}$, with $C_{2}^{\perp} \subseteq C_{1}$. The quantum codewords of the CSS code are superpositions of all classical codewords from the cosets of $C_{2}^{\perp}$ in $C_{1}$. Measuring the $Z$-type operators, therefore, corresponds to determining the error syndrome for $C_{1}$, whereas measuring the $X$-type operators determines the error syndrome for $C_{2}$. The usual 1-EPP protocol for correcting errors is for Bob to compute the difference, in both bases, between Alice's syndrome and his syndrome, and then to perform a Pauli operation to give his state the same syndromes as Alice's state. That is, Alice and Bob now each have a superposition over the same coset of $C_{2}^{\perp}$ within the same coset of $C_{1}$ (or rather, they have an entangled state, a superposition over all possible shared cosets for a given pair of syndromes). The decoding procedure then determines which coset of $C_{2}^{\perp}$ they share and uses that as the final decoded state.

More concretely, we can describe the classical procedure as follows: For the error correction stage, Alice computes and announces the parity checks for the code $C_{1}$. Bob subtracts his error syndrome from Alice's and flips bits (according to the optimal error-correction procedure) to produce a state with 0 relative error syndrome; that is, he should now have the same string as Alice. Then Alice and Bob perform privacy amplification: they compute the parity checks of $C_{2}^{\perp}$ (i.e., they multiply by the generator matrix of $C_{2}$ ) and use those as their final secret key bits.

There is one final step to convert the protocol to a "prepare-and-measure" protocol. Instead of preparing $N$ qubits and sending them to Bob, Alice prepares $2 N(1+\epsilon)$ (for BB84) or $3 N(1+\epsilon)$ (for the six-state scheme). Instead of waiting for Alice to announce which rotation she has performed $(I, H, T$, or $T^{2}$ ), Bob simply chooses one at random. Instead of rotating and then measuring in the $Z$ basis, Bob simply measures in the $X, Y$, or $Z$ basis, depending on which rotation he chose. Then Alice and Bob announce their bases, and discard those bits for which they measured different bases. With high probability, there will be at least $N$ remaining bits. Alice and Bob perform the error test on $m$ of them, and do error correction and privacy amplification on the remaining $N-m$. Since the discarded bits are just meaningless noise, they do not affect the security of the resulting "prepare-and-measure" protocol. The only difference is that security must now be measured in terms of the remaining bits rather than the original number of qubits sent. When we begin with a two-basis scheme, we end up with BB84; when we begin with a three-basis scheme, we end up with the six-state protocol.

\section{Difficulty in Generalization to Two-Way EPP's}

An obvious attempt to generalize Theorem 5 to 2-EPPs would be to simply use CSS-like (those with $X$-type and $Z$-type measurement operators only) 2-EPPs instead of CSS-like 1-EPPs. Unfortunately, this approach fails; another condition is needed.

For instance, consider the following 2-EPP, which we call EPP 1: Alice and Bob each measure $Z \otimes Z$ on pairs of EPR pairs. This can be implemented as a bilateral XOR: Alice performs an XOR from the first pair to the second, and Bob does the same. Then both Alice and Bob measure their qubit in the second pair and broadcast the measurement result. If Alice's and Bob's measurement outcomes disagree, they discard both pairs. On the other hand, if Alice's and Bob's measurement outcomes agree, then they keep the first pair for subsequent operations. Now, if there is exactly one bit-flip error between the two pairs, Alice and Bob will disagree; otherwise, they agree. Note that at most one EPR pair out of the original two would survive the measurement, but if Alice and Bob disagree, they discard both pairs. They do this for a large number of pairs; the surviving EPR pairs have a lower bit-flip error rate than the original ones.

Unfortunately, the surviving pairs also have a higher rate of phase errors, since phase errors propagate backward along a CNOT. Therefore, in the next round of the EPP, Alice and Bob measure $X \otimes X$ on pairs of EPR pairs. This can be implemented by performing a Hadamard transform, followed by the bilateral XOR and measurement described above. Alice and Bob should then perform another Hadamard to return the surviving EPR pair to its original basis. This procedure can detect the presence of a single phase error in the two pairs. If Alice and Bob discard EPR pairs for which their measurement results disagree, the surviving pairs will have a lower rate of phase errors than before.

The bit-flip error rate has increased again. However, the net effect of the two rounds taken together has been to decrease both the $X$ and $Z$ error rates (provided the error rates are not 


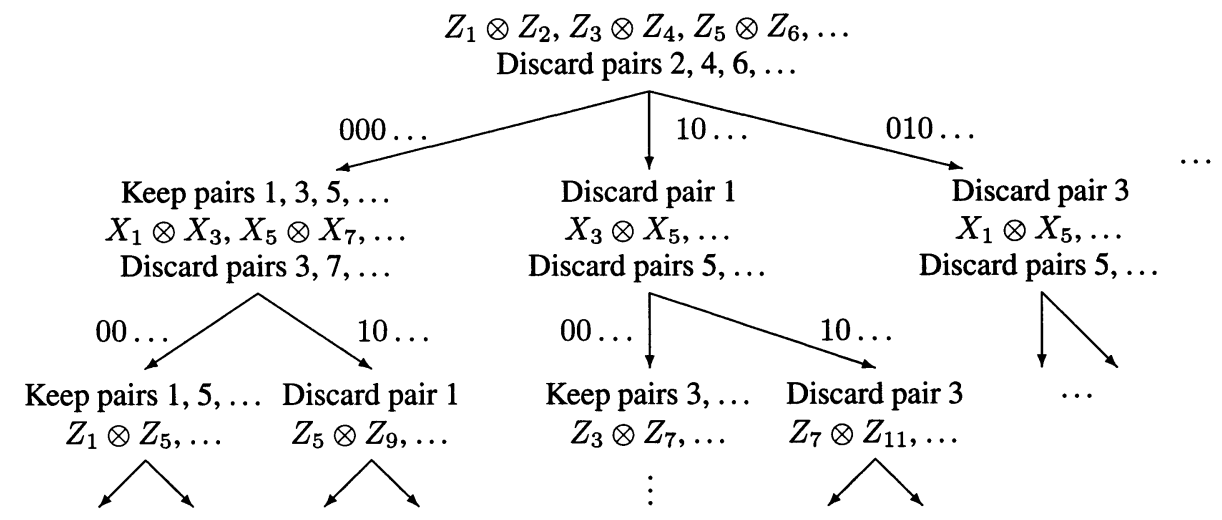

Fig. 4. Tree diagram for EPP 1.

too high to begin with). Alice and Bob can continue to repeat this procedure, measuring $X \otimes X$ alternately with $Z \otimes Z$, and the error rates will continue to improve. However, each round reduces the population of EPR pairs by at least half, so a better strategy is to switch to a more efficient 1-EPP once the error rates have dropped to the point where one is viable. Provided the initial error rate is not too large, this procedure eventually converges. The tree diagram for EPP 1 is given in Fig. 4.

The whole procedure only consists of measuring operators which are either $X$-type or $Z$-type, so the EPP is CSS-like. Still, we cannot convert this EPP to a "prepare-and-measure" BB84 QKD scheme.

What goes wrong? As is clear from Fig. 4, the EPP described is very definitely a 2-EPP, not a 1-EPP. In order to know which measurement to perform for the second round of the protocol, both Alice and Bob must know which EPR pairs survived the first round. Similarly, in the third round, they must know which EPR pairs survived the second round, and so forth.

In a "prepare-and-measure" scheme, Alice and Bob make all their measurements in the $Z$ basis, and ignore the $X$-basis parity checks because phase errors have no direct effect on the final key. They can, therefore, easily deduce the values of any operators which are the product of all Z's, but have no way of figuring out the measurement result for a product of all $X$ 's. Since the second round consists of measuring $X$ operators, Alice and Bob have no way of determining which bits to keep for the third round of the protocol, and, therefore, cannot complete the third round of the error-correction/detection process. That is, they do not know along which branch in the tree diagram they should proceed.

In a more intuitive language, the problem is that Alice and Bob do not have quantum computers in a prepare-and-measure protocol. Therefore, they cannot compute the phase error syndrome, which corresponds to the eigenvalues of the $X$-type operators. For this reason, they do not know which photons to throw away (conditional on the phase error syndrome) and cannot complete the $\mathrm{QKD}$ process.

\section{TWO-WAY QKD}

Having understood the failure of EPP 1, we now present a generalization of Theorem 5 .
Theorem 6-Main Theorem: Suppose a 2-EPP is CSS-like and also satisfies the following conditions.

1) The tree diagram only branches at $Z$-type operators, not at $X$-type operators.

2) The final decoding operations $U_{\mu}$ can depend arbitrarily on the outcome of the measured $Z$-type operators, but cannot depend on the outcomes of the measured $X$-type operators at all. The correction operation $P_{\mu}$ can depend on the outcome of $X$-type operators, but only by factors of $Z$.

Then the protocol can be converted to a "prepare-and-measure" QKD scheme with security similar to the EPP-based QKD scheme.

To understand these conditions, recall that the outcomes of $X$-type operators represent the phase error syndrome. Taken together, the two conditions say that the outcomes of $X$-type operators are used to perform phase error correction (by the factors of $Z$ in the correction operator $P_{\mu}$ ), but nothing else. For instance, no post-selection based on the phase error syndrome is allowed. From there, the intuition is identical to that for the proof of the Shor-Preskill result (Theorem 5). Phase errors do not affect the value of the key, so there is no need for Alice and Bob to compute the phase error syndrome at all. Therefore, Alice and Bob do not really need quantum computers and can execute a "prepare-and-measure protocol" instead.

The tree diagram of a 2-EPP satisfying the conditions of this theorem might look like the one depicted in Fig. 5. The "prepare-and-measure" protocol produced by this theorem has the following form.

1) Alice sends Bob $2 N(1+\epsilon)$ qubits, randomly choosing $|0\rangle$ or $|1\rangle$ for each and putting each in either the $X$ or $Z$ basis at random.

2) Bob chooses to measure each qubit in the $X$ or $Z$ basis at random.

3) Alice and Bob compare their measurement bases and discard those qubits for which the bases disagree. They keep $N$ remaining qubits.

4) Alice and Bob use $m$ of the qubits to estimate the error rate from the channel, getting values $p_{X}$ and $p_{Z}$. 


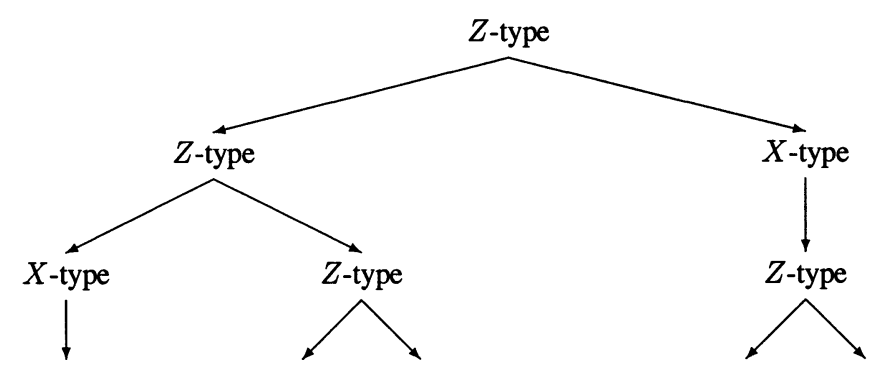

Fig. 5. Tree diagram of a 2-EPP satisfying the conditions of theorem 6.

5) They now perform a combination of classical two-way error detection/correction and classical privacy amplification based on the EPP. The outcomes of $Z$ 's serve two different functions: "advantage distillation" and also error correction. Indeed, Alice and Bob's ability to choose which branch to follow (e.g., which EPR pairs to keep or throw away) depending on the $Z$ operators means that Alice and Bob can perform error detection. Not necessarily all bit-flip errors are corrected. Since this is highly analogous to the "advantage distillation" procedure in classical cryptography, we will use the same name to denote such a procedure. In addition, the $Z$ operators measured in the EPP can also act as classical parity checks performed for error correction. Finally, the $X$ operators measured become parities extracted for privacy amplification. If $M_{\mu}$ is an $X$-type operator, let $v_{\mu}$ be a vector which is 1 for any coordinate where $M_{\mu}$ has an $X$, and is 0 for any coordinate where $M_{\mu}$ acts as the identity $I$. Consider the vector space $V$ generated by the $v_{\mu}$ 's for consecutive $X$-type operators. Then extract the parity for all vectors $u$ in the dual space $V^{\perp}$ of $V$. These become the bits used in the next round of error correction.

\section{ANOTHER TWO-WAY EPP}

Before proving the main theorem, we give an example of a 2-EPP that satisfies the conditions of Theorem. Like EPP 1, it will consist of alternating rounds of measurements designed to handle bit-flip errors ("B steps") and phase errors ("P steps").

B Step: A B step is just the same as the first round of EPP 1. Randomly permute all the EPR pairs. Afterwards, perform a bilateral XOR between pairs of EPR pairs, and measure one of the output pairs. This effectively measures the operator $Z \otimes Z$ for both Alice and Bob, and detects the presence of a single bit-flip error. Again, if Alice and Bob's measurement outcomes disagree, they discard the remaining EPR pair.

Note that this is similar to a classical protocol by Maurer for advantage distillation [29].

The second round must deal with phase errors; however, we will not be able to discard EPR pairs based on the result, since the conditions of the theorem bar us from altering our protocol based on the measurement results. Instead, we take inspiration from the classical repetition code.

A simple way to correct a single bit-flip error is to use the majority vote and encode the state $|0\rangle \mapsto|000\rangle,|1\rangle \mapsto|111\rangle$. Therefore,

$$
\alpha|0\rangle+\beta|1\rangle \mapsto \alpha|000\rangle+\beta|111\rangle
$$

Suppose the system is now corrupted by some bit-flip errors. A single bit-flip error can be detected by performing a majority vote. More precisely, one measures $Z_{1} Z_{2}$ to see if the first bit agrees with the second bit and also $Z_{1} Z_{3}$ to see if the first bit agrees with the third bit. These two measurements can be done coherently. The outcomes of the measurements are collectively called the error syndrome and can be used to correct the state coherently.

The three-qubit bit-flip error correction procedure can be turned into a three-qubit phase error correction procedure by simply applying the Hadamard transform, and into an EPP, following BDSW [3].

P Step: Randomly permute all the EPR pairs. Afterwards, group the EPR pairs into sets of three, and measure $X_{1} X_{2}$ and $X_{1} X_{3}$ on each set (for both Alice and Bob). This can be done (for instance) by performing a Hadamard transform, two bilateral XORs, measurement of the last two EPR pairs, and a final Hadamard transform. If Alice and Bob disagree on one measurement, Bob concludes the phase error was probably on one of the EPR pairs which was measured and does nothing; if both measurements disagree for Alice and Bob, Bob assumes the phase error was on the surviving EPR pair and corrects it by performing a $Z$ operation.

When there is only a single phase error among the three EPR pairs, this procedure outputs a single EPR pair with no phase error. However, when there are two or three phase errors, the final EPR pair always has a phase error. Therefore, when the phase error rate is low enough, iteration of this procedure will improve it indefinitely, while for higher phase error rates, the state will actually get worse.

The complete EPP protocol (EPP 2) consists of alternating B and $\mathrm{P}$ steps for a number of rounds, until the effective error rate has decreased to the point where 1-EPPs can take over. Then we decide on an appropriate CSS code and perform the corresponding 1-EPP. To get optimal performance, we should in fact use asymmetric CSS codes, which correct a fraction $f_{1}$ of bit flips and a different fraction $f_{2}$ of phase errors. Note that whenever $1-H\left(f_{1}\right)-H\left(f_{2}\right) \geq 0$, asymptotically, an asymmetric CSS code exists that will correct those fractions of errors with high fidelity. (A better bound might be obtained by considering the correlations between bit-flip and phase errors. See [22] for details.) We can view the whole EPP protocol as a kind of two-way concatenated code.

EPP 2 satisfies the conditions of Theorem 6: it is CSS-like, and measurements do not branch based on the outcome of $X$-type measurements (which only occur during $\mathrm{P}$ steps and in the final CSS code). Furthermore, we only do Pauli operations based on the outcome of $X$-type measurements. Thus, we can apply Theorem 6 to convert EPP 2 into the following "prepare-and-measure" QKD scheme.

\section{Protocol 2: Repeated Concatenation of BXOR With the Three-Qubit Phase Code}

1) Alice sends Bob a sequence of $N$ single photons as in either BB84 or the six-state scheme.

2) Alice and Bob sacrifice $m$ of those pairs to perform the refined data analysis. They abort if the error rates are too large. 
3) Alice and Bob randomly pair up their photons. Alice publicly announces the parity (XOR) of the bit values of each pair of her photons, say $x_{2 i-1} \oplus x_{2 i}$. Bob publicly announces the parity (XOR) of his corresponding pair of photons, say $y_{2 i-1} \oplus y_{2 i}$. If their parities agree, they keep one of the bits from the pair-i.e., Alice keeps $x_{2 i-1}$ and Bob keeps $y_{2 i-1}$. If their parities disagree, they throw away the whole pair. (This step comes from a B step.)

4) Alice and Bob randomly form trios of the remaining bits and compute the parity of each trio. They now regard those parities as their effective new bits. (This step comes from a P step.)

5) Steps 3) and 4) are repeated a prescribed number of times, say $r$, which depends on the error rate measured in step 2).

6) Alice and Bob randomly permute their pairs. They then apply a modified Shor and Preskill error correction/privacy amplification procedure. That is, Alice randomly picks a codeword $u$ in the code $C_{1}$ and broadcasts $u+w$ to Bob, where $w$ is her remaining bit string. Owing to the remaining noise in the channel, Bob's current bit string is instead $w+e$. He now adds $u+w$ to his string to obtain a corrupted string $u+e$. He can apply error correction for the code $C_{1}$ to recover $u$. Here we use a modified Shor and Preskill procedure that is based on an asymmetric CSS code that corrects up to a fraction $f_{1}$ of bit-flip errors and a different fraction, $f_{2}$, of phase errors.

7) Alice and Bob perform the coset extraction procedure to obtain the coset $u+C_{2}$, which gives their final key.

In order to determine if the resulting QKD protocol is secure or not at a given error rate, we need only study the behavior of EPP 2. Furthermore, by Lemmas 2 and 3 and the intervening discussion, we need only study the behavior of EPP 2 for uncorrelated Pauli channels with nice symmetry properties.

For the six-state scheme, this is completely straightforward: we just plug in the upper bounds on the error rates $\left(q_{X}, q_{Y}, q_{Z}\right)$ and see if EPP 2 converges. This upper bound on the error rates gives the worst case behavior. For the usual six-state scheme, we may even assume $q_{X}=q_{Y}=q_{Z}=q$. We can test for convergence with a simple computer program; we follow the error rates through B and $\mathrm{P}$ steps until they are small enough so that CSS coding is effective. If the program indicates convergence for $q$, the EPP definitely converges, and we have proved the six-state protocol is secure at bit error rate $q$. In this way, we have shown the six-state scheme remains secure to an error rate of at least $23.6 \%$. If the program does not converge, that does not necessarily imply that the six-state scheme is insecure using this post-processing method; it simply means it did not converge within the regime where our program is numerically reliable.

A study of BB84 is slightly more difficult. Alice and Bob do not know $\left(q_{X}, q_{Y}, q_{Z}\right)$, only $p_{X}=q_{Y}+q_{Z}$ and $p_{Z}=$ $q_{X}+q_{Y}$. There is one free parameter $q_{Y}=a$; then, for BB84, $q_{X}=q_{Z}=p-a$, where $p=p_{X}=p_{Z}$ is the bit error rate. To show that BB84 is secure using this post-processing scheme, we must show that EPP 2 converges for all values of $a \in[0, p]$. However, this is not immediately compatible with a numerical approach, since we would have to check infinitely many values of $a$. Instead, we first show analytically that $a=0$ (no $Y$ errors) gives the worst case; the proof is in Appendix III. Then we need only check in our program that EPP 2 converges for the uncorrelated Pauli channel $(p, 0, p)$. Our program then indicates that BB84 is secure to an error rate of at least $17.9 \%$.

It turns out, however, that alternating B and P steps is not optimal. EPPs based on other arrangements of these two steps can converge at higher error rates. For instance, for the three-basis protocol, we have discovered that a sequence of five B steps, followed by asymmetric CSS coding, converges to an error rate of at least $26.4 \%$, and that, therefore, the six-state scheme remains secure to at least this bit error rate. Similarly, setting $a=0$ in the two-basis protocol, a sequence of five B steps, followed by six P steps, followed by asymmetric CSS coding converges up to an error rate of at least $18.9 \%$. Since $a=0$ is again the worst case, this shows that BB84 can be secure to at least this bit error rate.

We remark that, in the preceding discussion, we have assumed that Alice and Bob simply throw away the error syndrome of each round immediately after its completion. Such an assumption greatly simplifies our analysis. However, in principle, Alice and Bob can employ an improved decoding scheme where they keep track of all the error syndromes and use them to improve the decoding in future rounds of the algorithm. It would be interesting to investigate in the future how much the tolerable error rates can be increased by such an improved decoding scheme. Of course, other improvements might be possible as well, including different kinds of $\mathrm{B}$ and $\mathrm{P}$ steps. The threshold error rate (i.e., the maximal bit error rate that can be tolerated) of a prepare-and-measure QKD scheme remains an important open question.

\section{Proof of the Main Theorem}

To prove Theorem 6, we begin with a QKD protocol using the 2-EPP directly. The security of this protocol follows immediately from Theorem 4. As in the Proof of Theorem 5, we then rearrange the protocol into a standard form in which it is clear that the $X$-type measurements are unnecesarry. From there, it is an easy step to a prepare-and-measure protocol.

1) Alice prepares $N$ EPR pairs. She performs a Hadamard transform on the second qubit for half of them, chosen at random.

2) Alice sends the second qubit from each EPR pair to Bob. Bob acknowledges receiving them, and then Alice tells him which ones have the Hadamard transform. Bob reverses all Hadamard transforms.

3) Alice and Bob select $m$ EPR pairs to test the error rate in the channel.

4) Alice and Bob perform the 2-EPP on the remaining $N-m$ EPR pairs. They now have a number of EPR pairs of very good fidelity.

5) Alice and Bob measure each remaining EPR pair in the $Z$ basis to produce a secure shared key. 


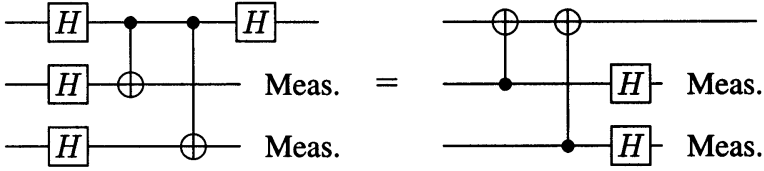

Fig. 6. Two equivalent ways to measure the operators $X_{1} X_{2}$ and $X_{1} X_{3}$.

The above protocol assumes a two-basis QKD scheme. For a three-basis scheme, Alice and Bob apply one of the three operations $I, T, T^{2}$ instead of $I$ or $H$.

To reduce the above EPP protocol to a prepare-and-measure one, we would like to eliminate the phase-error correction steps in the EPP protocol. For a CSS-like EPP, phase-error correction comes completely from the measurement of $X$-type operators $M_{\mu}$. We can perform such a measurement as a Hadamard transform, followed by a series of CNOTs with the same target qubit. Then we measure the target qubit, and Hadamard transform the others back to the original basis (see, for instance, the left network in Fig. 6). This procedure computes the parity of all the control qubits and the target qubit in the $X$ basis, and gives the eigenvalue of $M_{\mu}$. (Of course, in the context of an EPP, both Alice and Bob perform this procedure, and compare results.)

However, this series of gates, Hadamard, CNOT, Hadamard, is equivalent to a single CNOT gate with control and target reversed. This means, for example, that the two circuits depicted in Fig. 6 are mathematically equivalent. Note that the right-hand side depicts an essentially classical circuit composed of CNOTs (with a couple of $X$-basis measurements at the end). Instead of working with a quantum circuit for phase-error correction, as depicted by the left-hand side of the figure, one can work with the essentially classical circuit in the right-hand side.

The same principle holds in general. $X$-basis measurements can be written as effectively classical circuits consisting of a series of CNOTs (with the same control qubit but different target qubits), followed by a Hadamard transform and measurement on the control qubit. The qubits which survive the procedure have only experienced the CNOT gates. So it will be easy to convert this circuit to a truly classical one.

Note that each target qubit gets replaced by its XOR with the control qubit; in other words, by a parity which is orthogonal to the vector $v_{\mu}$ derived from $M_{\mu}$ by replacing $X$ 's with 1 's. For instance, in our sample EPP 2, we measure two $X$ operators in a row for a set of three qubits, $X_{1} X_{2}$ and $X_{1} X_{3}$. The effect of these measurements in the $Z$ basis is to map

$$
|a, b, c\rangle \mapsto|a+b, b, c\rangle \mapsto|a+b+c, b, c\rangle .
$$

That is, the first qubit gets replaced by the parity of all three qubits. We could also see this by noting that the only nontrivial vector which is orthogonal to both $(1,1,0)$ and $(1,0,1)$ is $(1,1,1)$.

However, for EPPs satisfying the conditions of Theorem 6, no choice of $M_{\nu}$ later in the protocol depends on the outcome of the $X$-type measurement $M_{\mu}$. Therefore, we can delay making the actual measurement until the end of the protocol, after we have measured all $Z$ operators. The EPP may call for correcting phase errors immediately by performing $Z$ rotations based on the measurement results, but we can delay those as well using the identities

$$
\begin{aligned}
& \mathrm{CNOT}(Z \otimes I)=(Z \otimes I) \mathrm{CNOT} \\
& \mathrm{CNOT}(I \otimes Z)=(Z \otimes Z) \mathrm{CNOT} .
\end{aligned}
$$

That is, we can move a $Z$ rotation from before a CNOT to after it, possibly at the price of having to do two of them instead. Ultimately, we end up with a circuit consisting only of $Z$-basis measurements and quantum CNOT gates (whose position may depend on the outcome of a $Z$ measurement), followed by $X$-basis measurements and phase shifts. This is an equivalent EPP to the one we began with.

In the QKD protocol, after performing the EPP, Alice and Bob measure each surviving EPR pair in the $Z$ basis to produce a key. But phase shifts are irrelevant if we are immediately going to measure in the $Z$ basis, so Alice and Bob need not actually perform them or the $X$-basis measurements controlling them.

Alice and Bob now have a completely classical circuit, followed by measuring all the qubits in the $Z$ basis. They get the same result if they instead measure all the qubits first, and then perform the classical circuit. The circuit they have is exactly the error correction and privacy amplification protocol described in Section VI as coming from the EPP. Note that any communication from Bob to Alice occurs during the classical circuit after the initial measurement.

To complete the transformation to a "prepare-and-measure" protocol, we follow a few additional steps from Shor and Preskill. Instead of preparing a number of EPR pairs and measuring them, Alice can just generate a random bit string, and send Bob the state he would have gotten if she made the EPR pairs and got that measurement result. That is, she sends Bob a series of 0's and 1's chosen at random, and puts half of them in the $X$ basis (when in the EPP protocol she would perform a Hadamard transform in Step 1), and puts half of them in the $Z$ basis (when there would be no Hadamard in the EPP protocol). Bob receives them, waits for Alice to tell him the basis, and then measures in that basis.

Of course, we can wait to decide on the EPP until after Bob receives his states, so it is equally good if Bob guesses a basis for each qubit and measures immediately. Then, when Alice tells Bob which basis she used, they discard any bits where the bases disagree. This gives the final "prepare-and-measure" protocol.

To prove the security of a six-state protocol, one uses three bases $X, Y$, and $Z$ in the appropriate place instead of just the $X$ and $Z$ bases. Otherwise, the proof is identical.

\section{CONCLUDING REMARKS}

We have proven the unconditional security of standard quantum key distribution schemes including BB84 and the six-state scheme. Our proof allows Alice and Bob to employ two-way classical communications. Compared to previous schemes, it has the advantage of tolerating substantially higher bit error rates. Indeed, we have shown that the BB84 scheme can be secure even at a bit error rate of $18.9 \%$ and the six-state scheme at $26.4 \%$. By tolerating such high bit error rates, our result may extend the distance of QKD and increase the key generation rate. Our result is conceptually interesting because it may spur progress in the study of 2-EPPs. We have introduced a 
new subclass of 2-EPPs and demonstrated that such a subclass of protocols can be reduced to standard BB84 and the six-state scheme. Our results demonstrate clearly that two-way classical communications can be used to enhance the secrecy capacity of a QKD scheme and also show the six-state scheme can intrinsically tolerate a higher bit error rate than BB84.

Our versions of the BB84 and six-state QKD schemes require two-way classical communications between Alice and Bob in the post-processing step of classical data (i.e., in the error correction and privacy amplification stage). This is not a bad thing in itself because any protocol of BB84 (or six-state) requires two-way classical communications anyway. Indeed, in the basis comparision step, Alice and Bob publicly announce their bases and throw away the polarization data that are transmitted and received in different bases. In order for both Alice and Bob to know which polarization data to keep, it is necessary to employ two-way classical communications. Of course, the "one-way" classical post-processing schemes require fewer rounds of communication (and, therefore, less time) to complete, so there appears to be a tradeoff between round complexity of the protocol and tolerable error rate.

Relating to earlier work on QKD, we remark that we have provided the first examples of unconditionally secure schemes for advantage distillation [29]-[31] in QKD. Finally, two-way entanglement purification techniques may provide a simple way to understand other security proofs. For instance, in Appendix IV, we provide a simple derivation of Inamori's security proofs [18], [19]. For future work, it would be interesting to take into account the effects of imperfections including faulty photon sources, lossy channels, and photon dark counts [20].

\section{APPENDIX I}

\section{PROOF OF LEMMA 1}

The statements that Alice and Bob will most likely share the same key and that the key is essentially random are clear. We will focus on proving the bound on Eve's information. The proof of this crucial part of Lemma 1 follows from the following two claims, which originally appeared in [23, supplementary Note II].

\section{Claim 7-High Fidelity Implies Low Entropy: If}

$$
\langle n \text { singlets }|\rho| n \text { singlets }\rangle>1-\delta
$$

where $\delta \ll 1$, then von Neumann entropy

$$
S(\rho)<-(1-\delta) \log _{2}(1-\delta)-\delta \log _{2} \frac{\delta}{\left(2^{2 R}-1\right)} .
$$

Proof: If $\langle n$ singlets $|\rho| n$ singlets $\rangle>1-\delta$, then the largest eigenvalues of the density matrix must be larger than $1-\delta$. The entropy of $\rho$ is, therefore, bounded above by that of

$\rho_{0}=\operatorname{diag}\left(1-\delta, \frac{\delta}{\left(2^{2 R}-1\right)}, \frac{\delta}{\left(2^{2 R}-1\right)}, \ldots, \frac{\delta}{\left(2^{2 R}-1\right)}\right)$.

That is, $\rho_{0}$ is diagonal with a large entry $1-\delta$ and with the remaining probability $\delta$ equally distributed between the remaining $2^{2 R}-1$ possibilities.

Claim 8-Entropy is a Bound to Mutual Information: Given any pure state $\left|\phi_{C D}\right\rangle$ of a system consisting of two subsystems $C$ and $D$, and any generalized measurements $X$ and $Y$ on $C$ and $D$, respectively, the entropy of each subsystem $S\left(\rho_{C}\right)$ (where
$\left.\rho_{C}=\operatorname{Tr}_{D}\left|\phi_{C D}\right\rangle\left\langle\phi_{C D}\right|\right)$ is an upper bound to the amount of mutual information between $X$ and $Y$.

Proof: This is a corollary to Holevo's theorem [17].

\section{APPENDIX II \\ PROOF OF LEMMA II}

We wish to show that, given any (not necessarily uncorrelated) Pauli channel, our procedure of testing the error rate and then choosing an appropriate code actually does correct the errors with high probability. The idea is that, because of the random permutation, the EPP treats symmetrically all errors with a given breakdown into $X, Y$, and $Z$ errors (the "type" of $P_{j}$ ). The type of the true error will be close to the estimated type. We then show that the EPP performs well for the likely types of error.

Since the channel is symmetric over all $N$ pairs, the pairs chosen for error testing are a representative sample, and the number of errors of any given kind in the sample will be close to the number of errors of the same kind in the remaining pairs. What we mean by the "same kind" bears a little explanation. As discussed before the statement of Lemma 3, we only directly measure the presence of two out of the three types of error, depending on which operation $\left(I, H, T\right.$, or $\left.T^{2}\right)$ we perform. For instance, when $I$ is performed, we measure the presence of only $X$ or $Y$ errors. However, since Eve has no knowledge of which operation is used for any particular qubit, the sample of test bits with a particular operation gives a good estimate of the number of the appropriate pair of errors in the remaining qubits of the sample. For instance, the fraction of errors among $I$ test qubits gives us a good estimate of the number of qubits with either $X$ or $Y$ errors in them. Then the deduced rates of $X, Y$, and $Z$ errors (as discussed before Lemma 3 ) give a good estimate of the actual error rates in the untested pairs.

For any particular instance of the protocol, the channel performs a particular $N$-qubit Pauli operation $P_{j}$ (with probability $q_{j}$ ). For any particular $j$, let $q_{i}^{d}$ be the deduced fraction of errors of type $i(i=X, Y, Z)$ in the sample and let $q_{i}^{u}$ be the actual fraction of errors of type $i$ in the untested pairs ("d" for "deduced" and "u" for "untested"). Then for large $N$, with high probability

$$
\left|q_{i}^{\mathrm{d}}-q_{i}^{\mathrm{u}}\right|<\epsilon
$$

(That is, the deduced error rate is close to the true error rate.) Naturally, $q_{i}^{\mathrm{d}}$ and $q_{i}^{\mathrm{u}}$ will depend on $j$, but we suppress this dependence to simplify the notation.

Let us now restrict attention to one particular set of values for $q_{i}^{\mathrm{d}}$ and $q_{i}^{\mathrm{u}}$ (which need not be equal, but which satisfy condition (5)). If the $q_{i}^{\mathrm{d}}$ are large, Alice and Bob will abort the protocol. Otherwise, we wish to show that the EPP used by Alice and Bob will correct most errors with these parameters.

To see this, we note that the EPP will correct the uncorrelated Pauli channel $\left(q_{X}^{\mathrm{u}}, q_{Y}^{\mathrm{u}}, q_{Z}^{\mathrm{u}}\right)$ on $N-m$ EPR pairs with high fidelity $F$. Suppose the EPP gives fidelity $F_{j}$ whenever the $N$-qubit Pauli operation $P_{j}$ occurs (for a stabilizer EPP, $F_{j}$ will be either 0 or 1 ). Then

$$
F=\sum_{j} p_{j} F_{j}
$$


where $p_{j}$ is the probability of the Pauli operation $P_{j}$ for the uncorrelated Pauli channel (not the true channel). We can break the sum over $j$ into two parts. The first part will consist of the set $S$ of $j$ for which $P_{j}$ contains exactly $n_{X}=q_{X}^{\mathrm{u}}(N-m) X$ errors, $n_{Y}=q_{Y}^{\mathrm{u}}(N-m) Y$ errors, and $n_{Z}=q_{Z}^{\mathrm{u}}(N-m) Z$ errors (the $n_{i}$ are integers by the definition of $q_{i}^{\mathrm{u}}$ ). The second part consists of all other $j$. Now, let $p$ be the probability of any particular error in $S$, so

$$
\sum_{j \notin S} p_{j} F_{j} \leq \sum_{j \notin S} p_{j}=1-\sum_{j \in S} p
$$

so

$$
\begin{aligned}
F & =\sum_{j \in S} p_{j} F_{j}+\sum_{j \notin S} p_{j} F_{j} \\
& \leq p \sum_{j \in S} F_{j}+1-p|S| \\
& =1-p|S|\left(1-\sum_{j \in S} F_{j} /|S|\right) .
\end{aligned}
$$

But

$$
p=\left(q_{X}^{\mathrm{u}}\right)^{n_{X}}\left(q_{Y}^{\mathrm{u}}\right)^{n_{Y}}\left(q_{Z}^{\mathrm{u}}\right)^{n_{Z}}\left(q_{I}^{\mathrm{u}}\right)^{n_{I}}
$$

where $q_{I}^{\mathrm{u}}=1-\left(q_{X}^{\mathrm{u}}+q_{Y}^{\mathrm{u}}+q_{Z}^{\mathrm{u}}\right)$ is the probability of identity operations, and $n_{I}=q_{I}^{\mathrm{u}}(N-m)$ is the actual number of identity operations. $S$ contains $(N-m) ! /\left(n_{X} ! n_{Y} ! n_{Z} ! n_{I} !\right)$ elements, so using Stirling's approximation, we find

$$
p|S| \approx\left(\frac{2 \pi}{N-m}\right)^{3 / 2} \frac{1}{\sqrt{q_{X}^{u} q_{Y}^{u} q_{Z}^{u} q_{I}^{u}}} .
$$

This is only polynomially small in $N-m$. In order for $F$ to be exponentially close to 1 in (10), we therefore require that $\sum_{j \in S} F_{j}$ be $[1-\exp (-O(N))]|S|$.

Now we can approximate the fidelity of the EPP for the general Pauli channel $\left(\mathcal{P}_{i}, q_{i}\right)$. We again write $F=\sum q_{i} F_{i}$ (with the same $F_{i}$ 's, which only depend on the EPP, not the channel), and recall that $q_{i}=q_{j}=q_{n_{X}, n_{Y}, n_{Z}}$ whenever $i$ and $j$ have the same numbers $\left(n_{X}, n_{Y}, n_{Z}\right)$ of $X, Y$, and $Z$ errors. That means we can write

$$
F=\sum_{n_{X}, n_{Y}, n_{Z}} q_{n_{X}, n_{Y}, n_{Z}} \sum_{i \in S_{n_{X}, n_{Y}, n_{Z}}} F_{i} .
$$

But, except with exponentially small probability, the values $n_{X}$, $n_{Y}, n_{Z}$ are within the allowed $\epsilon$-sized window for the EPP, which we have shown means that

$$
\sum_{i \in S} F_{i}=[1-\exp (-O(N))]|S| .
$$

Thus,

$$
\begin{aligned}
F=\sum_{n_{X}, n_{Y}, n_{Z}} q_{n_{X}, n_{Y}, n_{Z}}|S|[1-\exp (-O(N))] \\
-\exp (-O(N)) .
\end{aligned}
$$

Since

$$
\sum_{n_{X}, n_{Y}, n_{Z}} q_{n_{X}, n_{Y}, n_{Z}}|S|=1
$$

it follows that the fidelity for the general Pauli channel is exponentially close to 1 .

\section{APPENDIX III \\ ProOf That $a=0$ IS THE Worst CASE}

In this appendix, we will show that it is sufficient to check the $a=0$ case (with no $Y$ errors) when determining convergence of the 2-EPPs we study for the BB84 protocol.

Theorem 9: Suppose an EPP starts with a B step, followed by any series of B and/or P steps, followed by asymmetric CSS coding. Suppose $0 \leq p<1 / 4$. If the EPP converges for the uncorrelated Pauli channel $(p, 0, p)$, then it will also converge for all uncorrelated Pauli channels $(p-a, a, p-a)$, with $0 \leq$ $a \leq p$.

The initial condition $p<1 / 4$ simply ensures that (for any value of $a$ ) the state is more likely to be correct than incorrect, and will be satisfied easily by all parameter sets we consider. In fact, when $p \geq 1 / 4$, an intercept-resend attack defeats BB84 (see Section II).

Proof: To do this, we will need to look at the behavior of the three error rates as we perform steps of the protocol. After each B or P step, there is a new set of effective error rates on the pairs surviving the round.

It is worth noting two things about protocols of the given form. First, if the initial density matrix comes from a Pauli channel, then the effective channel after any number of rounds will also be a Pauli channel. This is because all operations are from the Clifford group, which preserves the Pauli group. Second, if the initial channel causes errors which are uncorrelated between EPR pairs, this property will also be preserved after an arbitrary number of $\mathrm{B}$ and $\mathrm{P}$ rounds. This is because both $\mathrm{B}$ and $\mathrm{P}$ rounds keep at most one of the pairs which interact, so there is no opportunity to create correlations between pairs which survive to the next round. Therefore, we can completely describe the effective error rates at any given point in the protocol by a triplet $\left(q_{X}, q_{Y}, q_{Z}\right)$.

Suppose we start with error rates $\left(q_{X}, q_{Y}, q_{Z}\right)$ and perform a B step. Given any of the 16 possible configurations of errors, we can deduce whether the remaining pair is discarded, and if not, whether it has an error, and what kind of error it is. The new error rates on the surviving pairs are then $\left(q_{X}^{\prime}, q_{Y}^{\prime}, q_{Z}^{\prime}\right)$

$$
\begin{aligned}
q_{X}^{\prime} & =\left(q_{X}^{2}+q_{Y}^{2}\right) / p_{S} \\
q_{Y}^{\prime} & =2 q_{X} q_{Y} / p_{S} \\
q_{Z}^{\prime} & =2\left(1-q_{X}-q_{Y}-q_{Z}\right) q_{Z} / p_{S} \\
p_{S} & =1-2\left(q_{X}+q_{Y}\right)\left(1-q_{X}-q_{Y}\right)
\end{aligned}
$$

where $p_{S}$ is the probability that a pair will survive the check.

If we have error rates $\left(q_{X}, q_{Y}, q_{Z}\right)$ and perform a $\mathrm{P}$ step, we get new error rates $\left(q_{X}^{\prime}, q_{Y}^{\prime}, q_{Z}^{\prime}\right)$

$$
\begin{aligned}
q_{X}^{\prime} & =3 q_{I}^{2}\left(q_{X}+q_{Y}\right)+6 q_{I} q_{X} q_{Z}+3 q_{X}^{2} q_{Y}+q_{X}^{3} \\
q_{Y}^{\prime} & =6 q_{I} q_{Y} q_{Z}+3 q_{X}\left(q_{Y}^{2}+q_{Z}^{2}\right)+3 q_{Y} q_{Z}^{2}+q_{Y}^{3} \\
q_{Z}^{\prime} & =3 q_{I}\left(q_{Y}^{2}+q_{Z}^{2}\right)+6 q_{X} q_{Y} q_{Z}+3 q_{Y}^{2} q_{Z}+q_{Z}^{3} \\
q_{I} & =1-q_{X}-q_{Y}-q_{Z}
\end{aligned}
$$

where $q_{I}$ is the initial probability of no error. 
To prove the theorem, we change variables. Instead of working with $\left(q_{X}, q_{Y}, q_{Z}\right)$, we will work with $\left(p_{Z}, p_{X}, \Delta\right)$

$$
\begin{aligned}
p_{Z} & =q_{X}+q_{Y} \\
p_{X} & =q_{Y}+q_{Z} \\
\Delta & =q_{Z}-q_{Y}=p_{X}-2 a .
\end{aligned}
$$

As $a$ increases, $p_{X}$ and $p_{Z}$ stay the same, while $\Delta$ decreases. We will show that the protocol behaves worse for larger $\Delta$, so the worst case is $a=0$.

In the new variables, a $\mathrm{B}$ step maps the error rates from $\left(p_{Z}, p_{X}, \Delta\right)$ to $\left(p_{Z}^{\prime}, p_{X}^{\prime}, \Delta^{\prime}\right)$

$$
\begin{aligned}
p_{Z}^{\prime} & =p_{Z}^{2} / p_{S} \\
p_{X}^{\prime} & =\left[p_{X}-p_{X}^{2}+\Delta\left(1-2 p_{Z}-\Delta\right)\right] / p_{S} \\
\Delta^{\prime} & =\left[p_{X}\left(1-2 p_{Z}\right)+\Delta\left(1-2 p_{X}\right)\right] / p_{S} \\
p_{S} & =1-2 p_{Z}+2 p_{Z}^{2} .
\end{aligned}
$$

Since $p_{X}, p_{Z}<1 / 2$ always in the regime of interest, $\Delta^{\prime}$ is increasing in $\Delta$, and $p_{Z}^{\prime}$ never depends on $\Delta$ at all. Provided $1-2 p_{Z}-2 \Delta>0, p_{X}^{\prime}$ also increases with $\Delta$. When this is true, $\Delta^{\prime}$ and $p_{X}^{\prime}$ also both increase with $p_{X}$.

A $\mathrm{P}$ step takes the error rates from $\left(p_{Z}, p_{X}, \Delta\right)$ to $\left(p_{Z}^{\prime}, p_{X}^{\prime}, \Delta^{\prime}\right)$ with the following relations:

$$
\begin{aligned}
p_{Z}^{\prime} & =3 p_{Z}\left(1-p_{Z}\right)^{2}+p_{Z}^{3} \\
p_{X}^{\prime} & =3 p_{X}^{2}\left(1-p_{X}\right)+p_{X}^{3} \\
\Delta^{\prime} & =3 \Delta^{2}\left(1-2 p_{Z}-\Delta\right)+\Delta^{3} .
\end{aligned}
$$

This time, $p_{X}^{\prime}$ and $p_{Z}^{\prime}$ only depend on $p_{X}$ and $p_{Z}$, respectively, never on $\Delta . p_{X}^{\prime}$ increases with $p_{X} . \Delta^{\prime}$ only depends on $\Delta$ and $p_{Z}$, and increases with $\Delta$ if two conditions $-1-2 p_{Z}-\Delta>0$ and $\Delta \geq 0$ - are simultaneously satisfied.

Claim 10: The following inequalities hold:

1) at all points after the initial B step, $\Delta \geq 0$;

2) $1-2 p_{Z}-2 \Delta>0$ always.

Note that when $p_{X}+p_{Z}<1 / 2$, so that at least half the time there is no error, it follows that $1-2 p_{Z}-2 \Delta>0$, since $\Delta<p_{X}$. However, it is not clear if the condition $p_{X}+p_{Z}<1 / 2$ is preserved under the $\mathrm{B}$ and $\mathrm{P}$ steps.

From this claim, the theorem will follow: consider running the protocol starting with error rates $\left(p_{Z}, p_{X}, \Delta\right)=(p, p, p)$ or $\left(p, p, \Delta_{0}\right)$, with $\Delta_{0}<p$. Since the value of $p_{Z}$ at any given time only depends on the previous value of $p_{Z}, p_{Z}$ will always be equal in these two cases. At any time, $p_{X}$ for the first case will be greater than or equal to $p_{X}$ for the second case, and $\Delta$ for the first case will be greater than or equal to $\Delta$ for the second case. This is true by induction: it is true initially, and at all steps, $p_{X}^{\prime}$ and $\Delta^{\prime}$ increase with $p_{X}$ and $\Delta$ from the previous step. Thus, the worst case is when $\Delta=p$, which means $a=0$.

Proof (of Claim): Immediately after the initial B step, $\Delta^{\prime} \geq 0$, because in this step, $p_{X}=p_{Z}=p$ by the symmetry of BB84, and $\Delta \geq-p$. After subsequent B steps, $\Delta^{\prime} \geq 0$ if $\Delta \geq 0$, since $1-2 p_{X}$ and $1-2 p_{Z}$ are always positive.

After a $\mathrm{P}$ step, $\Delta^{\prime} \geq 0$ if $3\left(1-2 p_{Z}-2 / 3 \Delta\right) \geq 0$. This will immediately follow if we can show $1-2 p_{Z}-2 \Delta>0$, since before a $\mathrm{P}$ step, $\Delta \geq 0$ always. Then, by induction, we will have shown $\Delta \geq 0$ at all points after the initial B step.
Now, after a B step

$$
\begin{aligned}
1-2 p_{Z}^{\prime}-2 \Delta^{\prime} & =\left[1-2 p_{Z}^{2}-2 p_{X}\left(1-2 p_{Z}\right)-2 \Delta\left(1-2 p_{X}\right)\right] / p_{S} \\
& =\left[\left(1-2 p_{Z}-2 \Delta\right)\left(1-2 p_{X}\right)+2 p_{Z}\left(1-p_{Z}\right)\right] / p_{S} .
\end{aligned}
$$

The first term is always positive, so the sum is clearly positive as well when $1-2 p_{Z}-2 \Delta>0$.

After a P step

$$
1-2 p_{Z}^{\prime}=\left(1-2 p_{Z}\right)^{3}
$$

so

$$
\begin{aligned}
1-2 p_{Z}^{\prime}-2 \Delta^{\prime}= & \left(1-2 p_{Z}\right)^{3}-6 \Delta^{2}\left(1-2 p_{Z}\right)+4 \Delta^{3} \\
= & \left(1-2 p_{Z}-2 \Delta\right) \\
& \cdot\left[\left(1-2 p_{Z}\right)^{2}+2 \Delta\left(1-2 p_{Z}-\Delta\right)\right] .
\end{aligned}
$$

Again, this is positive when $1-2 p_{Z}-2 \Delta>0$ and $\Delta \geq 0$. This proves the claim and the theorem.

\section{APPENDIX IV \\ INAMORI'S SECURITY PROOFS}

In this appendix, we provide a simple derivation of Inamori's proofs of BB84 and the six-state scheme and discuss why our protocols can tolerate a higher rate than his.

Inamori's protocols require two-way communications. His protocol can be rephrased as follows.

1) Alice and Bob are assumed to share initially a random string and the goal of QKD is to extend this string. Alice and Bob also choose a classical error-correcting code $C_{1}$.

2) Alice sends Bob a sequence of single photons as in either BB84 or the six-state scheme.

3) They throw away all polarization data that are prepared in different bases and keep only the ones that are prepared in the same bases.

4) They randomly select $m$ of those pairs and perform a refined data analysis to find out the error rate of the various bases.

5) Alice measures the remaining $N-m=s$ particles to generate a random string $v$. Since $v$ is a random string, it generally has nontrivial error syndrome when regarded as a corrupted state of the codeword of $C_{1}$. Alice transmits that error syndrome in an encrypted form to Bob. This is done by using a one-time pad encryption with (part of) the common string they initially share as the key.

6) Bob corrects his error to recover the string $v$.

7) Alice and Bob discard all the bits where they disagree and keep only the ones where they agree.

8) Alice and Bob now perform privacy amplification on the remaining string to generate a secure string.

We remark that Inamori's protocol is, in fact, a simple errorcorrection scheme and satisfies the conditions of Theorem 6. Therefore, it is convenient to study it using the language of 2-EPPs introduced in the current extended abstract. 


\section{A. BB84 With Inamori's Protocol}

Let us now consider the efficiency of BB84 based on Inamori's protocol. Suppose the error rate of each basis is found to be $p$ in Step 4). Now, in Step 5) above, Alice and Bob have to sacrifice a pre-shared secret key whose length must be at least the size of the error syndrome of an $s$-bit string. In other words, the length of the pre-shared secret key used up by Alice and Bob is at least

$$
l_{s a c}=s h(p)
$$

bits where $h(x)=-x \log _{2} x-(1-x) \log _{2}(1-x)$.

What is the length of the key they generate from the process? Recall that in Step 7), Alice and Bob discard all the bits where they disagree and keep only the ones where they agree. The length of their reconciled key is, therefore, given by the number of bits where Alice and Bob agree. In other words, Alice and Bob generate a reconciled key of the length

$$
r=s(1-p) \text {. }
$$

Since Eve may have some partial information on the reconciled key, Alice and Bob have to sacrifice some of the reconciled key for privacy amplification. Let us consider privacy amplification. For BB84, the worst case density matrix is again of the form

$$
\operatorname{diag}(1-2 p, p, p, 0)
$$

in the Bell-basis using the convention in [3].

In Step 7), Alice and Bob post-select only the bits where they agree. With such post-selection, the (unnormalized) conditional density matrix becomes

$$
\operatorname{diag}(1-2 p, 0, p, 0) .
$$

In other words, the phase error rate is

$$
\frac{p}{(1-2 p+p)}=\frac{p}{1-p} \text {. }
$$

Therefore, Alice and Bob must sacrifice a further fraction

$$
f_{B B 84}=h\left(\frac{p}{1-p}\right)
$$

of their reconciled key for privacy amplification.

In summary, the length of the reconciled key is $r=s(1-p)$, as given by (40), ff which, a fraction $h\left(\frac{p}{1-p}\right)$ has to be consumed for privacy amplification. Therefore, the final key generated by Alice and Bob is of length $\left[1-h\left(\frac{p}{1-p}\right)\right] s(1-p)$. In addition, from (39), a length of $l_{s a c}=s h(p)$ of a pre-shared secret key has to be consumed. Therefore, the net key generation rate is given by

$$
\begin{aligned}
{\left[1-h\left(\frac{p}{1-p}\right)\right] } & s(1-p)-s h(p) \\
& =s(1-p)\left[1-h\left(\frac{p}{1-p}\right)-\frac{h(p)}{1-p}\right] .
\end{aligned}
$$

From (45), one can conclude that in Inamori's protocol, the net key generation rate is positive provided that

$$
1-h\left(\frac{p}{1-p}\right)-\frac{h(p)}{1-p}>0
$$

which is exactly what appears just before [18, Sec. 5].
Note that, for BB84, the maximal tolerable error rate of Inamori's scheme is actually worse than in Shor-Preskill.

\section{B. Six-State Scheme With Inamori's Protocol}

Let us now consider the six-state scheme. Suppose that in Step 4), the error rate is found to be $p$. In Step 5), the length of the pre-shared key sacrificed by Alice and Bob is the same as in BB84 and is given by (39). Also, the length of the reconciled key is the same as in BB84 and is given by (40).

Here is the key difference between the six-state scheme and BB84: For the six-state scheme, there is more symmetry. In particular, as discussed in Section IV-B, for an EPP that corresponds to the six-state scheme, one only needs to consider a depolarizing channel. The density matrix is

$$
\operatorname{diag}(1-3(p / 2), p / 2, p / 2, p / 2) \text {. }
$$

On post-selecting the bits where Alice and Bob agree, the (unnormalized) density matrix becomes

$$
\operatorname{diag}(1-3(p / 2), 0, p / 2,0) .
$$

Therefore, the post-selected phase error rate is

$$
\frac{p / 2}{1-3(p / 2)+p / 2}=\frac{p}{2(1-p)} \text {. }
$$

Comparing (43) and (49), we see that a big difference between BB84 and six-state in the Inamori's protocol is that the post-selected phase error rate for the six-state is only half of that for BB84. Consequently, Alice and Bob sacrifice fewer bits for privacy amplification in the six-state case. In fact, only a smaller fraction, namely, a fraction

$$
f_{\text {six }}=h\left(\frac{p}{2(1-p)}\right)
$$

of the reconciled key needs to be sacrificed in the privacy amplification process.

In summary, the length of the reconciled key is $r=s(1-p)$, as given by (40). Of which, from (50), only a fraction $h\left(\frac{p}{2(1-p)}\right)$ has to be consumed for privacy amplification. Therefore, the final key generated by Alice and Bob is of length $\left[1-h\left(\frac{p}{2(1-p)}\right)\right] s(1-p)$. In addition, from (39), a length of $l_{\text {sac }}=\operatorname{sh}(p)$ of a pre-shared secret key has to be consumed. Therefore, the net key generation rate is given by

$$
\begin{aligned}
& {\left[1-h\left(\frac{p}{2(1-p)}\right)\right] s(1-p)-s h(p)} \\
& =s(1-p)\left[1-h\left(\frac{p}{2(1-p)}\right)-\frac{h(p)}{1-p}\right] .
\end{aligned}
$$

From (51), one can conclude that in Inamori's protocol for the six-state scheme, the net key generation rate is positive provided that

$$
1-h\left(\frac{p}{2(1-p)}\right)-\frac{h(p)}{1-p}>0
$$

which is precisely what Inamori gave in the equation just under [19, Property 1 on p. 3]. Comparing (46) and (52), one can see that the key difference between BB84 and six-state for Inamori's protocol is in the second term of the expressions. In the case 
of the six-state scheme, there is an extra factor of 2 in the denominator inside the entropy function. As noted before, this is because the six-state scheme has more symmetry and gives a lower phase error rate (upon post-selection of bits where Alice and Bob do agree) than BB84.

From (52), Inamori's protocol for the six-state case can tolerate a bit error rate of roughly $12.6 \%$. A more recent protocol [22] for the six-state scheme can tolerate a marginally higher bit error rate and, unlike Inamori's scheme, it requires only one-way classical post-processing. We remark that the six-state scheme with our Protocol 2 tolerates a much higher error rate (about $23 \%$, or as high as $26.4 \%$ varying the sequence of $\mathrm{B}$ and $\mathrm{P}$ steps) than a six-state scheme with Inamori's protocol.

\section{ACKNOWLEDGMENT}

The authors wish to particularly thank Jayson Cohen for his generous help in Matlab programming, and David DiVincenzo and Debbie Leung for suggesting the tree diagram representation of an EPP. Enlightening discussions and references on bounds on error rates in prior art QKD schemes have been kindly provided by Norbert Lütkenhaus and Nicolas Gisin. They have also greatly benefitted from helpful communications with Nabil Amer, Charles Bennett, Hitoshi Inamori, Ueli M. Maurer, Renato Renner, John Smolin, Barbara Terhal and, particularly, John Preskill and Peter W. Shor. This research was conducted in part during the period that D. Gottesman served as a Clay Long-Term CMI Prize Fellow and during a visit of H.-K. Lo at the California Institute of Technologyy.

\section{REFERENCES}

[1] H. Bechmann-Pasquinucci and N. Gisin, "Incoherent and coherent eavesdropping in the 6-state protocol of quantum cryptography," Phys. Rev., vol. A59, p. 4238, 1999.

[2] C. H. Bennett and G. Brassard, "Quantum cryptography: Public key distribution and coin tossing," in Proc. IEEE Int. Conf. Computers, Systems, and Signal Processing. New York: IEEE Tress, 1984, p. 175.

[3] C. H. Bennett, D. P. DiVincenzo, J. A. Smolin, and W. K. Wootters, "Mixed state entanglement and quantum error correction," Phys. Rev., vol. A54, p. 3824, 1996.

[4] M. Ben-Or. Presentation at, for example, Introductory Workshop in Quantum Computation. [Online]http://zeta.msri.org/calendar/talks/ TalkInfo/1416/show_talk

[5] E. Biham, M. Boyer, P. O. Boykin, T. Mor, and V. Roychowdhury, "A proof of the security of quantum key distribution," in Proc. 32nd Annu. ACM Symp. Theory of Computing (STOC). New York: ACM Press, 2000 , p. 715.

[6] D. Bruss, "Optimal eavesdropping in quantum cryptography with six states," Phys. Rev. Lett., vol. 81, p. 3018, 1998.

[7] I. Cirac and N. Gisin, "Coherent eavesdropping strategies for the 4-state quantum cryptography protocol," Phys. Lett., vol. A229, p. 1, 1997.

[8] D. Deutsch, A. Ekert, R. Jozsa, C. Macchiavello, S. Popescu, and A. Sanpera, "Quantum privacy amplification and the security of quantum cryptography over noisy channels," Phys. Rev. Lett., vol. 77, p. 2818, 1996. Erratum in Phys. Rev. Lett., vol. 80, p. 2022, 1998.

[9] D. P. DiVincenzo, P. W. Shor, and J. A. Smolin, "Quantum channel capacity of very noisy channels," Phys. Rev. A, vol. 57, p. 830, 1998. Also, [Online]. Available: http://xxx.lanl.gov/abs/quant-ph/9706061.
[10] A. K. Ekert, "Quantum cryptography based on Bell's theorem," Phys. Rev. Lett., vol. 67, p. 661, 1991.

[11] C. Fuchs, N. Gisin, R. B. Griffiths, C. S. Niu, and A. Peres, "Optimal eavesdropping in quantum cryptography. I," Phys. Rev., vol. A56, p. $1163,1997$.

[12] N. Gisin, G. Ribordy, W. Tittel, and H. Zbinden, "Quantum cryptography," Rev. Mod. Phys., vol. 74, pp. 145-195. Also, [Online]. Available: http://xxx.lanl.gov/abs/quant-ph/0101098.

[13] D. Gottesman. An introduction to quantum error correction. [Online] Available: http://xxx.lanl.gov/abs/quant-ph/0004072.

[14] D. Gottesman and H.-K. Lo, "From quantum cheating to quantum security," Phys. Today, vol. 53, no. 11, p. 22, Nov. 2000. Also, [Online]. Available: http://www.physicstoday.org/pt/vol-53/iss-11/p22.html.

[15] D. Gottesman and J. Preskill, "Secure quantum key distribution using squeezed states," Phys. Rev., vol. A63, p. 22309, 2001.

[16] F. Grosshans and P. Grangier. Reverse reconciliation protocols for quantum cryptography with continuous variables. [Online]. Available: http://xxx.lanl.gov/abs/quant-ph/0204127.

[17] A. S. Holevo, "Bounds for the quantity of information transmitted by a quantum communication channel," Probl. Inform. Transm. (U.S.S.R.), vol. 9, p. 177, 1973.

[18] H. Inamori. Security of EPR-based quantum key distribution (Los Alamos e-print archive). [Online]. Available: http://xxx.lanl. gov/abs/quant-ph/0008064.

[19] - Security of EPR-based quantum key distribution using three bases (Los Alamos e-print archive). [Online]. Available: http://xxx.lanl. gov/abs/quant-ph/0008076.

[20] H. Inamori, N. Lütkenhaus, and D. Mayers. Unconditional security of practical quantum key distribution. (Los Alamos e-print archive). [Online]. Available: http://xxx.lanl.gov/abs/quant-ph/0107017.

[21] A. Lamas-Linares, C. Simon, J. C. Howell, and D. Bouwmeester, "Science," report, (DOI:10.1126/science.1068972).

[22] H.-K. Lo, "Proof of unconditional security of six-state quantum key distribution scheme," Quant. Inform. Comput. (QIC), vol. 1, no. 2, pp. 81-94, 2001. Also, [Online]. Available: http://xxx.lanl.gov/abs/quant$\mathrm{ph} / 0102138$.

[23] H.-K. Lo and H. F. Chau, "Unconditional security of quantum key distribution over arbitrarily long distances," Science, vol. 283, pp. 2050-2056, 1999. Also, [Online]. Available: http://xxx.lanl.gov/abs/quant$\mathrm{ph} / 9803006$.

[24] _ "Quantum cryptographic system with reduced data loss," U.S. Patent 5732 139, Mar. 24, 1998.

[25] H.-K. Lo, H. F. Chau, and M. Ardehali. Efficient quantum key distribution scheme and proof of its unconditional security (Los Alamos e-print archive. [Online]. Available: http://xxx.lanl.gov/abs/quant-ph/0011056

[26] H.-K. Lo, S. Popescu, and T. Spiller, Introduction to Quantum Computation and Information. Singapore: World Scientific. Hardcover 1998, paperback 2001, $364 \mathrm{pp}$.

[27] N. Lütkenhaus, "Estimates for practical quantum cryptography," Phys Rev., vol. A59, p. 3301, 1999.

[28] M. A. Nielsen and I. L. Chuang, Quantum Computation and Quantum Information. Cambridge, U.K.: Cambridge Univ. Press, 2000. 676 pp.

[29] U. M. Maurer, "Protocols for secret key agreement by public discussion based on common information," in Advances in Cryptology-Proc. Crypto' 92. New York: Springer-Verlag, 1993, vol. 740, pp. 461-470.

[30] — "Secret key agreement by public discussion from common information," IEEE Trans. Inform. Theory, vol. 39, p. 733, 1993.

[31] U. M. Maurer and S. Wolf, "Unconditionally secure key agreement and intrinsic information," IEEE Trans. Inform. Theory, vol. 45, pp. 499-514, Mar. 1999.

[32] D. Mayers, "Unconditional security in Quantum Cryptography," $J$. Assoc. Comput. Mach., vol. 48, no. 3, pp. 351-406. Also, [Online]. Available: http://xxx.lanl.gov/abs/quant-ph/9802025.

[33] P. W. Shor and J. Preskill, "Simple proof of security of the BB84 quantum key distribution protocol," Phys. Rev. Lett., vol. 85, p. 441, 2000. Also, [Online]. Available: http://xxx.lanl.gov/abs/quant$\mathrm{ph} / 0003004$

[34] S. Wiesner, “Conjugate coding," Sigact News, vol. 15, no. 1, p. 78, 1983.

[35] D. Mayers, "Quantum key distribution and string oblivious transfer in noisy channels," in Advances in Cryptology-Proc. Crypto'96. New York: Springer-Verlag, 1996, p. 343. 